\title{
1 Emergent RNA-RNA interactions can promote stability in a nascent
}

\section{2 phototrophic endosymbiosis}

3 Benjamin H. Jenkins ${ }^{1,2 *}$, Finlay Maguire ${ }^{3}$, Guy Leonard ${ }^{1,2}$, Joshua D. Eaton ${ }^{1}$, Steve West ${ }^{1}$,

4 Benjamin E. Housden ${ }^{1}$, David, S. Milner ${ }^{1,2}$, \& Thomas A. Richards ${ }^{1,2}$ *

$5 \quad{ }^{1}$ Living Systems Institute and Biosciences, University of Exeter, Devon EX4 4QD, UK

$6 \quad{ }^{2}$ Department of Zoology, University of Oxford, 11a Mansfield Road, Oxford OX1 3SZ, UK

$7{ }^{3}$ Faculty of Computer Science, Dalhousie University, 6050 University Ave, Halifax NS B3H

8 1W5, Canada

9 *Correspondence: thomas.richards@zoo.ox.ac.uk \& ben.jenkins@zoo.ox.ac.uk

\section{KEYWORDS}

11 Paramecium bursaria, algae, mutualism, symbiosis, RNAi, Dicer, RDR, siRNA

\section{ABSTRACT}

13 Eukaryote-eukaryote endosymbiosis was responsible for the spread of chloroplast (plastid)

14 organelles. Stability is required for the metabolic and genetic integration that drives the

15 establishment of new organelles, yet the mechanisms which act to stabilise nascent

16 endosymbioses - between two fundamentally selfish biological organisms - are unclear.

17 Theory suggests that enforcement mechanisms, which punish misbehaviour, may act to

18 stabilise such interactions by resolving conflict. However, how such mechanisms can emerge

19 in a nascent endosymbiosis has yet to be explored. Here, we propose that endosymbiont-

20 host RNA-RNA interactions, arising from digestion of the endosymbiont population, can

21 result in a cost to host growth for breakdown of the endosymbiosis. Using the model

22 nascent endosymbiosis, Paramecium bursaria - Chlorella spp., we demonstrate that this

23 mechanism is dependent on the host RNA-interference (RNAi) system. We reveal through

24 small RNA (SRNA) sequencing that endosymbiont-derived mRNA released upon

25 endosymbiont digestion can be processed by the host RNAi system into 23-nt sRNA. We

26 predict multiple regions of shared sequence identity between endosymbiont and host mRNA, and demonstrate through delivery of synthetic endosymbiont sRNA that exposure to these regions can knock-down expression of complementary host genes, resulting in a cost to host growth. This process of host gene knock-down in response to endosymbiont-derived RNA processing by host RNAi factors, which we term 'RNAi-collisions', represents a 
31 mechanism which can promote stability in a nascent eukaryote-eukaryote endosymbiosis.

32 By imposing a cost for breakdown of the endosymbiosis, endosymbiont-host RNA-RNA

33 interactions may drive maintenance of the symbiosis across fluctuating ecological conditions

34 and symbiotic status.

\section{SIGNIFICANCE STATEMENT}

36 Stable endosymbiosis between eukaryotic microbes has driven the evolution of further

37 cellular complexity. Yet the mechanisms which can act to stabilise a nascent eukaryote-

38 eukaryote endosymbiosis are unclear. Using the model nascent endosymbiotic system,

39 Paramecium bursaria-Chlorella, we demonstrate that endosymbiont-host RNA-RNA

40 interactions can drive a cost to host growth upon endosymbiont digestion, punishing the

41 host for misbehaviour. These RNA-RNA interactions are facilitated by the host RNA-

42 interference system. For endosymbiont mRNA sharing a high-level of sequence identity with

43 host transcripts, this process can result in host gene knock-down. We propose that these

44 endosymbiont-host RNA-RNA interactions-'RNAi collisions'-represent a viable enforcement

45 mechanism to sanction the host for breakdown of the endosymbiosis, promoting the

46 stability of a nascent endosymbiotic interaction.

\section{MAIN TEXT}

48 A nascent endosymbiosis between eukaryotes was a pre-requisite for the spread of 49 photosynthetic organelles, such as plastids ${ }^{1-5}$. This transition from transiently engulfed cells

50 to obligate organelles is driven by metabolic and genetic integration. Yet, to become 51 manifest, a stable intermediary state must exist upon which this process of integration can 52 proceed $^{1,3,5-8}$. Conflict is an inevitable outcome of all symbioses, the resolution of which can 53 significantly impact the stability of an interaction. Enforcement mechanisms which punish 54 misbehaviour can act to stabilise symbioses ${ }^{9,10}$, yet we know little about how these can 55 emerge in a nascent endosymbiotic interaction. Paramecium bursaria - a ciliate protist 56 which harbours a clonal population of intracellular green algae, Chlorella spp. ${ }^{11-13}$ 57 represents a tractable model system to study emergent mechanisms in a nascent 58 endosymbiosis $^{14}$. The interaction is facultative ${ }^{11,14-17}$ and based on two-way metabolic 59 exchange ${ }^{18-25}$. Endosymbiotic algae are housed within modified host phagosomes called 60 perialgal vacuoles, which may be fused with host lysosomes to trigger digestion ${ }^{26}$ allowing $P$. 61 bursaria to maintain control over the interaction in the event of conflict ${ }^{27-29}$. However, it is 
62 unclear how this endosymbiotic system is protected from over-exploitation by the host

63 which would ultimately lead the interaction to collapse $e^{30-35}$.

64 RNA-RNA interactions can play a role in host-pathogen symbiotic systems ${ }^{36-40}$, 65 whereby RNA can 'hi-jack' the small interfering RNA (siRNA) pathway of the symbiotic 66 partner to modulate expression of genes involved in virulence or alternatively resistance.

67 Whether analogous RNA-RNA interactions could occur in an endosymbiotic system has yet 68 to be elucidated. The presence of a functional siRNA pathway in Paramecium, and its role in 69 RNA-interference (RNAi), has been validated as a tool for gene silencing in both Paramecium 70 bursaria $^{41}$ and non-photo-endosymbiotic congener, Paramecium tetraurelia ${ }^{42-46}$. RNAi can

71 be initiated through the provision of bacterial food transformed to express double-stranded 72 RNA (dsRNA) with high sequence similarity to a target transcript ${ }^{41,43}$. Paramecium can also process single-stranded RNA (ssRNA) - including ribosomal RNA (rRNA) and messenger RNA (mRNA) - derived from a prokaryotic cell acquired through phagotrophy ${ }^{42}$. This processing is mediated by conserved RNAi protein components which also function in endogenous transcriptome regulation ${ }^{41,42,44,47}$. Significantly, these studies propose that mRNA, derived both exogenously ${ }^{41,42}$ and endogenously ${ }^{41,44}$, can act as substrates for siRNA generation in Paramecium.

We demonstrate that RNA released upon digestion of the algal endosymbiont is processed by the host RNAi system in P. bursaria. For endosymbiont-derived mRNA sharing a high-level of sequence identity with host transcripts, this processing may interfere with endogenous host gene expression resulting in a cost to host growth (Fig. S1). We track the interaction through sRNA sequencing, recapitulate the effect through exposure to synthetic endosymbiont RNA, and demonstrate that this mechanism is mediated by host Dicer, Piwi, Pds1 and RdRP proteins. This process of host gene knock-down in response to endosymbiont-derived RNA processing by host RNAi factors, which we term 'RNAicollisions', represents a candidate enforcement mechanism which can promote stability in a nascent eukaryote-eukaryote endosymbiosis. By imposing a cost for breakdown of the endosymbiosis, endosymbiont-host RNA-RNA interactions may drive maintenance of the symbiosis across fluctuating ecological conditions and symbiotic status.

91 Endosymbiont digestion in P. bursaria results in an RNAi-mediated 'physiological cost' to 
93 P. bursaria can be purged of endosymbiotic algae via treatment with the ribosomal

94 translational inhibitor, cycloheximide ${ }^{14}$. A comparison of ribosomal protein (RP) L29A

95 predicted protein sequences, the active site of cycloheximide function, confirmed that

96 Paramecium possess a specific nucleotide polymorphism identified as a determinant of

97 cycloheximide resistance in other species (Fig. S2). This substitution was absent in all green

98 algal species assessed, including known algal endosymbionts of $P$. bursaria. Upon treatment

99 with cycloheximide, a significant reduction in algal-chlorophyll fluorescent intensity per host

100 cell was observed after two-to-three days (Fig. 1A). A clear de-coupling of host $P$. bursaria

101 cell number and algal fluorescence was also observed, consistent with translational

102 inhibition in the algae but not the host (Fig. S2), demonstrating that loss of a photosynthetic

103 endosymbiont population does not immediately result in a decline in host cell number.

104 Additional staining with LysoTracker Green to identify acidic vacuoles, including lysosomes,

105 revealed that this loss of algal fluorescence was linked to increased lysosomal activity in the

106 host cytoplasmic environment (Fig. 1B). These data support that elimination of the

107 endosymbiotic algal population during cycloheximide treatment ${ }^{14,26}$ is triggered by host

108 digestion.

109 Continued treatment with cycloheximide resulted in a significant retardation to $P$.

110 bursaria culture growth, however this same effect was not observed in the non-photo-

111 endosymbiotic congener species, P. tetraurelia (Fig. 1C). Both Paramecium species harbour

112 the same cycloheximide resistance conferring substitution in RPL29A (Fig. S2), suggesting

113 that elimination of endosymbiotic algae is costly to $P$. bursaria culture growth. Loss of algal

114 derived photosynthate and plastid derived metabolites represents an obvious cost.

115 However, we sought to explore whether a part of this cost may be attributed to host

116 exposure to endosymbiont-derived RNA during digestion of the endosymbiont population,

117 and whether this cost was mediated by the host RNAi system.

118 Knock-down of Dcr1 (a host-encoded endoribonuclease Dicer required for siRNA 119 generation) through complementary dsRNA exposure significantly rescued the cost to $P$. 120 bursaria culture growth associated with cycloheximide treatment (Fig. 1D and Fig. S3). This 121 was consistent with Dcr1 (Dicer) knock-down in a prior study attenuating the effect of E. coli 122 vector-based RNAi feeding in $P$. bursaria ${ }^{41}$. In the aforementioned study, knock-down of 123 host-encoded PiwiA1, PiwiC1 (AGO-Piwi effectors required for targeted RNA cleavage) and 
124 Pds1 (a Paramecium-specific component with an unknown but essential role in exogenously

125 induced RNAi) also attenuated an E. coli-vector feeding-based RNAi effect ${ }^{41}$. Here, knock-

126 down of host-encoded PiwiA1 and Pds1 similarly rescued the cost to $P$. bursaria culture

127 growth associated with cycloheximide treatment (Fig. 1E and Fig. S3). Involvement of Pds1

128 confirms that the observed RNAi effect is mediated by the host, as no identifiable

129 homologue of Pds1 could be identified in the green algal genomes and transcriptomes

130 sampled $^{41,48}$. Furthermore, it excludes the possibility that off-target effects arising from host

131 Dicer knock-down, including potential compensatory function of additional Dicer or Dicer-

132 like paralogues in P. bursaria ${ }^{41}$, could be responsible for the RNAi effect observed. The near

133 complete rescue observed upon partial knock-down of Dicer suggests that host Dicer

134 processing, rather than loss of algal derived metabolites, is a more significant factor relating

135 to growth retardation under these conditions. Taken together, these data suggest that the

136 physiological cost to $P$. bursaria growth incurred during cycloheximide treatment, in which

137 the endosymbiotic algae are being broken down and digested by the host, is mediated by

138 host-encoded RNAi components. These data support the occurrence of RNAi-mediated RNA-

139 RNA interactions between endosymbiont and host.

140 Endosymbiont breakdown triggers an abundance of Dicer-dependent endosymbiont 141 derived SRNA within $P$. bursaria

142 To investigate the occurrence of RNA-RNA interactions between endosymbiont and host, we

143 tracked the abundance of endosymbiont-derived, host-processed SRNA in $P$. bursaria during

144 endosymbiont digestion. Disruption of host RNAi was achieved through partial knock-down

145 of Dicer, allowing us to directly test for an increase in endosymbiotic algal-derived 23-nt

146 sRNA (the size associated with host Dicer processing ${ }^{41,42,49}$ ) resulting from cycloheximide

147 induced endosymbiont breakdown. Upon treatment with cycloheximide, we identified an

148 increased abundance in all 21-29 nt reads mapping to endosymbiont-derived mRNA over

149 two-to-three days (Fig. 2A and Fig. S4). This same trend was observed for reads mapping to

150 algal endosymbiont rRNA-derived sRNA (Fig. S5). Reads were mapped with 100\% identity,

151 allowing no mismatches. Any reads that additionally mapped to the host with $100 \%$ identity

152 were removed, to ensure that the subset of SRNA detected was of definitive algal origin. A

153 significant increase in algal mRNA-derived 23-nt sense and antisense sRNA demonstrates a

154 greater abundance of potential RNAi substrates during endosymbiont digestion ${ }^{41,42,49}$. 
155 Partial knock-down of host Dicer during cycloheximide treatment significantly ablated 156 this endosymbiont mRNA-derived 23-nt antisense abundance after three days of 157 cycloheximide treatment (Fig. 2B and Fig. S4). This suggests that an increase in 158 endosymbiont mRNA-derived 23-nt antisense sRNA during endosymbiont digestion is 159 dependent on host Dicer function. This is consistent with knock-down of host Dicer 160 specifically reducing $23-n t$ sRNA abundance in Paramecium ${ }^{41,42,49}$, and the observation that 161 Paramecium RNAi factors are capable of processing both endogenously and exogenously 162 derived $\mathrm{mRNA}^{42,44}$. It is important to note that this effect likely under-represents the full 163 extent of host Dicer processing, due to the requirement for a paradoxical and therefore 164 incomplete Dicer perturbation through Dicer-dependent RNAi-based knock-down ${ }^{41}$. 165 Furthermore, these data represent only a subset of the sRNA potentially present due to the 166 stringent mapping approach used to identify endosymbiont-derived SRNA, as any reads that 167 additionally mapped to host RNA template with $100 \%$ sequence identity in either 168 orientation were excluded from this analysis. While these reads with high shared sequence 169 identity are the most important subset of sRNA for the identification of putative mRNA170 mRNA interactions between endosymbiont and host, their exclusion here has allowed us to 171 identify host RNAi processing of definitively endosymbiont-derived transcripts. Interestingly, 172 one of the host-derived transcripts that mapped with $100 \%$ identity to an excluded 173 endosymbiont-derived 23-nt sRNA was P. bursaria heat shock protein 90 (HSP90). An 174 investigation of regions of high shared sequence identity between endosymbiont and host is 175 explored below.

176 Next, we conducted a series of control observations. Firstly, we assessed whether 177 cycloheximide treatment was altering general host-derived SRNA production, despite the 178 inferred host resistance discussed above (Fig. S2). Reads were mapped to a dataset of 20 179 host transcripts that contained no potential 23-nt overlap with any identified algal 180 transcripts (allowing for $\leq 2-n t$ mismatches), to ensure that these host transcripts were 181 unaffected by an increased rate of putative RNA-RNA interactions derived from the 182 endosymbiont (Fig. S6). No significant increase in 23-nt abundance was observed for reads 183 mapping to this subset of host mRNA transcripts during cycloheximide treatment, consistent 184 with host resistance to cycloheximide not altering the host-derived population of sRNAs 185 with low sequence identity to algal mRNA. Secondly, we assessed whether endosymbiotic 
algal strains cultured under free-living conditions would also generate an abundance of 23-

187 nt sRNA upon cycloheximide treatment (Fig. S7). Importantly, no clear increase in 21-25 nt

188 algal-derived SRNA abundance was observed during algal treatment with cycloheximide

189 when grown outside of the host cytoplasmic environment, nor was a 23-nt peak in algal

190 derived sRNAs evident. This is consistent with data from a previous study which found that

191 the algal endosymbiont of $P$. bursaria was not actively generating sRNA $>20-n t^{41}$. These

192 results suggest that increased algal endosymbiont-derived 23-nt sRNA abundance upon

193 treatment with cycloheximide is dependent on host sRNA processing within the

194 endosymbiotic system. Taken together, these data indicate that an abundance of

195 endosymbiont-derived RNAs are released during endosymbiont digestion, which then act as

196 substrate for processing by the host RNAi system, resulting in 23-nt sRNAs. Importantly,

197 processing of endosymbiont-derived SRNA in a host Dicer-dependent manner indicates that

198 these interactions are occurring in the host cytoplasm, supporting the hypothesis that

199 endosymbiont-host mRNA-mRNA interactions are possible.

200 Comparison of transcriptome data reveals the potential for host transcript interaction by 201 endosymbiont-derived RNAs

202 To understand the extent of possible RNA-RNA interactions between endosymbiont and

203 host, we built a bioinformatic transcriptome processing tool, eDicer

204 (https://github.com/fmaguire/eDicer), which allows identification of all possible Dicer-

205 generated sense and antisense oligonucleotides produced from a given transcriptome

206 dataset. By mapping 23-nt reads to a second ('host') dataset allowing for $\leq 2-n t$ mismatches,

207 potential RNA-RNA interactions between an input (endosymbiont, vector, or food) and a

208 host RNA population can be identified. Inclusion of reads with $\leq 2$-nt mismatches were based

209 on the tolerance for mismatching complementarity reported during RNAi-mediated knock-

210 down of gene expression in multiple systems ${ }^{50-54}$. Confirmation of a similar mismatch

211 tolerance in P. bursaria is shown in Fig. 3E \& Fig. S12. For a full overview of the eDicer

212 comparative analysis process, please refer to the Supplementary Methods.

213 Using a dataset consisting of transcripts binned as either 'endosymbiont' or 'host'

214 (using a curated P. bursaria transcriptome ${ }^{55}$ ), we identified 35,703 distinct 23-nt putative

215 mRNA-mRNA interactions between the $P$. bursaria 'host' and 'endosymbiont' RNA

216 populations, representing $0.121 \%$ of the total inventory of distinct host 23 -nt k-mers 
217 identified (Fig. S8A; see also Table S2 \& Supplementary Methods). This was found to be

218 120-fold greater than the number of putative 23-nt mRNA-mRNA interactions predicted

219 between bacterial food and host transcripts (from two different bacterial sources).

220 Furthermore, the ratio of total:'potentially-lethal' putative 23-nt mRNA-mRNA interactions

221 (determined by cross-referencing genes known to be conditionally essential in

222 Saccharomyces cerevisiae ${ }^{56}$ ) was found to be greater between endosymbiont-and-host

223 transcripts (1:1.4) than between bacterial food-and-host transcripts (1:0.16/0.17). Similar

224 patterns were observed for predicted rRNA-rRNA interactions (Fig. S8B, see also Table S2 \&

225 Supplementary Methods). These in silico analyses demonstrate that there is far greater

226 potential for the occurrence of both mRNA-mRNA and rRNA-rRNA interactions between the

227 algal endosymbiont and host (eukaryote-eukaryote) RNA populations, than there is between

228 bacterial food and host (prokaryote-eukaryote) RNA populations.

229 In vivo exposure to a synthetic endosymbiont mRNA-derived chimera generates knock-

230 down of high-identity host transcripts

231 Having identified the occurrence of putative endosymbiont-host RNA-RNA interactions in

232 silico, we assessed whether exposure to synthetic fragments of algal mRNA could

233 recapitulate the cost to host growth observed during endosymbiont digestion (Figure 1E).

234 We identified ten endosymbiont mRNA interaction fragments that shared $>91 \%$ (or $21-n t$ )

235 sequence identity with host transcripts across a 23-nt region, selected at random from the

236 analysis reported in Fig. S8A. Each mRNA interaction fragment was chosen to contain at

237 least 1 SNP specific to the endosymbiont to ensure that any identifiable effect could be

238 attributed to an endosymbiont-like transcript, rather than the host version. These

239 interaction fragments were predicted to still be effective RNAi templates for $P$. bursaria

240 based on the tolerance for mis-matching complementarity observed in RNAi-mediated

241 knock-down in other systems ${ }^{50-54}$ (and confirmed below in P. bursaria; Fig. 3E \& Fig. S12).

242 Six mRNA interaction fragments showed putative homology to 'non-lethal' yeast genes

243 (including EF1- $\alpha$ and HSP9O); two showed putative homology to 'lethal' yeast genes

244 (including tub- $\beta$ ); and the remaining two had no identifiable homologues in yeast (Table S1).

245 Significantly, HSP9O was the host transcript previously identified as a candidate for putative

246 endosymbiont-host RNA-RNA interaction in the sRNA analysis of Fig. 2. However, to ensure

247 that any identifiable effect could be attributed to the endosymbiont-derived transcript, the 
248 newly identified interaction fragment was chosen from a different region of this gene 249 containing 2 SNPs specific to the endosymbiont. All ten mRNA interaction fragments were 250 composed of the predicted interacting 23-nt sRNA sequence (Fig. S8A) flanked by 11-nt of 251 contiguous 'non-hit' endosymbiont transcript as a filler on each side (Table S3). These ten 252 45-nt fragments were combined into a single 450-nt synthetic endosymbiotic algal chimera 253 (Fig. 3A), cloned into an L4440 plasmid, and transformed into E. coli for feeding-induced 254 RNAi.

255 Exposure to endosymbiotic algal chimera dsRNA resulted in significant retardation of 256 P. bursaria culture growth (Fig. 3B). Once more, this effect was attenuated by knock-down 257 of host Dicer, demonstrating an RNAi-mediated response to synthetic endosymbiont-host 258 mRNA-mRNA interactions that resembled the host response to endosymbiont digestion in 259 Fig. 1C-E. It is important to note that synthetic exposure to endosymbiont-derived RNA via 260 an E. coli feeding vector would likely over-represent these putative RNA interactions. 261 However, to address this issue, we designed a non-hit 'nonsense' control composed of a 262 tandem assembly of the 11-nt regions of contiguous 'non-hit' algal transcript present in the 263 chimera. A relative dilution of endosymbiont chimera dsRNA delivery alongside the 264 'nonsense' control (1,1:1 and 1:3) also resulted in $P$. bursaria culture growth retardation 265 (Fig. S9), demonstrating that a reduced relative delivery of synthetic endosymbiont RNA can 266 also result in a cost to host growth. Similar experiments were conducted to investigate the 267 possibility of endosymbiont-host rRNA-rRNA interactions, but showed that there was no 268 equivalent effect on host growth, suggesting that ribosomal RNA is shielded from such 269 effects (Fig. S10, see also Table S4).

270 To identify which putative mRNA-mRNA interactions were capable of facilitating a cost 271 to $P$. bursaria culture growth, we identified the ten 450-nt host transcripts predicted to be 272 hit by each endosymbiont derived 23-nt mRNA interaction fragment used in the chimera 273 (Figure 3C - three examples are shown). Individual exposure to dsRNA corresponding to 274 each individual but longer $P$. bursaria transcript-form identified three which resulted in a 275 significant cost to host growth, relative to Dicer knock-down controls (Fig. 3D; see Fig. S11 276 for all 10). These P. bursaria transcripts correspond to elongation factor-1 $\alpha(E F-1 \alpha)$, heat 277 shock protein 90 (HSP90; the host transcript identified as a candidate for putative 278 endosymbiont-host RNA-RNA interaction in the sRNA analysis of Fig. 2 ), and tubulin- $\beta$ chain 
$279(t u b-\beta)$. It can therefore be inferred that these are the 23-nt mRNA interaction fragments

280 (Fig. 3A/C) that likely resulted in a cost to host growth observed during synthetic 281 endosymbiont chimera dsRNA exposure (Fig. 3B). Using mRNA extracted from P. bursaria 282 during chimera-RNAi feeding described above (Fig. 3B), qPCR revealed a reduction in host 283 transcript expression of $E F-1 \alpha$ and $t u b-\beta$ in response to endosymbiont chimera dsRNA 284 exposure (Fig. 3E). Host transcript expression of HSP9O appears inconclusive, however, 285 expression of all three host transcripts was partially rescued upon knock-down of host Dicer 286 (Fig. S12). From these data we can therefore conclude that $23-n t$ of $>91 \%$ complementary 287 'endosymbiont' transcript is sufficient to facilitate detectable knock-down of a 288 corresponding host transcript, demonstrating that exposure to endosymbiont-derived RNA 289 originating from phagosomes is capable of impacting host gene expression via host RNAi 290 knock-down.

291 It is important to consider that delivery of a synthetic endosymbiont-derived RNA 292 chimera represents only an approximation of the putative 23-nt RNA-RNA interactions 293 occurring in P. bursaria. Exposure to endosymbiont RNA via an E. coli feeding vector would 294 likely over-represent these putative interactions. However, the random generation of 23-nt 295 Dicer substrates across the dsRNA chimera (only three regions of which resulted in a 296 detectable cost to host growth; Fig. 3A/D \& Fig. S11) represents only part of the wider $E$. 297 coli-derived RNA population processed by the host RNAi system during chimera-RNAi 298 feeding. Together with dilution of endosymbiont chimera dsRNA delivery (Fig. S9), these 299 observations support the hypothesis that only a relatively small number of 23-nt RNA-RNA 300 interactions can induce a cost to host growth in $P$. bursaria. Furthermore, these data 301 confirm that sRNAs with $\leq 2-n t$ mismatches are effective templates for RNAi-mediated 302 knock-down of gene expression in P. bursaria, suggesting that the sRNA analysis in Fig. 2 303 may under-represent the true scale of endosymbiont-derived sRNAs that can result in RNA304 RNA interactions between endosymbiont and host. Importantly, we note that the 305 occurrence of putative endosymbiont-host RNA-RNA interactions does not exclude a wider 306 cost to host growth arising from processing a large population of algal derived RNAs with no 307 defined host target, a process which must pose a cost to host cellular economics and 308 transcriptional control/fidelity. For further justification of this 'synthetic' approach, and a 309 consideration of the results that we can reliably draw from these data, see Discussion S1. 
310 Single-stranded delivery of synthetic endosymbiont-derived RNA, analogous to 311 endosymbiont mRNA, results in a cost to $P$. bursaria growth

312 As RNA derived naturally from the endosymbiont was unlikely to be double-stranded, two

313 further constructs were designed to assess the efficacy of synthetic endosymbiont chimera 314 single-stranded RNA (sSRNA) exposure in P. bursaria (Fig. 4 \& Fig. S13). Exposure to 315 endosymbiont chimera ssRNA also resulted in a significant cost to $P$. bursaria culture 316 growth, however this effect was only observed when delivered in the sense orientation $317([+]$ ssRNA). Notably, chimera [+]ssRNA exposure resulted in a greater cost to host growth 318 than chimera dsRNA exposure. A similar orientation bias was observed upon Dicer knock319 down during cycloheximide treatment in a prior experiment, in which delivery of Dicer $320[+]$ ssRNA rescued culture growth to a greater extent than delivery of Dicer [-]ssRNA or Dicer dsRNA (Fig. S14). Importantly, the orientation of [+]ssRNA represents the same orientation as the mRNA transcripts from which each of these target templates were identified.

Knock-down of host Dicer (dsRNA delivery) was able to attenuate the cost to host 324 growth associated with endosymbiont chimera [+]ssRNA exposure (Fig. 4). Importantly, 325 simultaneous knock-down of host Rdr1 and Rdr2 (RNA-dependent RNA polymerases 326 involved in amplification of primary or secondary sRNA triggers during $\mathrm{RNAi}^{42,46}$ ) also 327 significantly rescued this cost to host growth. This is consistent with the role of RdRP 328 proteins in processing exogenous SRNA, partially degraded mRNA cleavage products, or full329 length mRNA transcripts in Paramecium ${ }^{42,44-46}$. As for Dicer, knock-down of host RdRP 330 through RNAi represents a potential paradox, and so constitutes only partial or transient 331 loss of function ${ }^{41}$. However, we are again confident that disruption in this manner would be 332 sufficient to perturb RdRP-mediated RNAi in P. bursaria after 14 days, as seen in Fig. 4. 333 While the mechanism of down-stream Dicer processing of RdRP-generated dsRNA 334 substrates remains unknown (see Discussion S1), we can nonetheless infer from these data 335 that single-stranded RNA-induced RNAi knock-down in P. bursaria is partially dependent 336 upon host RdRP function.

337 Irrespective of the mechanistic basis for a sense oriented ssRNA knock-down bias, it is 338 important to note that delivery of chimera [+]ssRNA demonstrated here represents the 339 same orientation as the endosymbiont derived mRNA interaction fragments from which 340 these putative endosymbiont-host RNA-RNA interactions were identified. This is consistent 
341 with the observation that Paramecium RNAi factors are capable of processing both

342 endogenously and exogenously derived $\mathrm{mRNA}^{42,44}$. The ability to facilitate a cost to host

343 growth through delivery of single-stranded RNA, in the same orientation as the host target

344 mRNA, therefore supports the hypothesis that a cost to host growth upon endosymbiont

345 digestion can be facilitated by host RNAi-mediated RNA-RNA interactions between

346 endosymbiont and host mRNA populations.

\section{DISCUSSION}

348 Through manipulation of the $P$. bursaria - Chlorella spp. endosymbiotic system, we have 349 demonstrated that RNA released upon digestion of the algal endosymbiont is processed by 350 the host RNAi system. For endosymbiont-derived mRNA sharing a high-level of sequence 351 identity with host transcripts, this processing can result in knock-down of endogenous host 352 gene expression, resulting in a cost to host growth (Fig. S1). We therefore postulate that 353 these RNA-RNA interactions are of importance in eukaryote-eukaryote endosymbioses 354 where partners are likely to share greater sequence similarity, especially among conserved 355 transcripts which tend to be more highly represented among lethal or conditionally 356 essential genes ${ }^{56}$. Due to the inherent difficulty in characterising such mechanisms directly, 357 we have relied on a multiple experimental approach to demonstrate the viability of putative 358 RNA-RNA interactions at each stage of the process. We have tracked the interaction through 359 sRNA sequencing, recapitulated the effect through exposure to synthetic endosymbiont360 derived RNA - including sense ssRNA analogous to endosymbiont mRNA - and 361 demonstrated that this mechanism is mediated by host Dicer, Piwi, Pds1 and RdRP proteins.

362 This process of host gene knock-down in response to endosymbiont-derived RNA processing 363 by host RNAi factors, which we term 'RNAi-collisions', represents a candidate enforcement 364 mechanism to sanction the host for breakdown of the interaction, a factor that would 365 promote stability in a nascent eukaryote-eukaryote endosymbiosis.

366 The long-term maintenance of symbiotic interactions represents a quandary for 367 evolutionary theory ${ }^{30-35}$. How do such relationships avoid over-exploitation by one partner 368 that would ultimately lead the interaction to collapse? Partner switching is one option, 369 however the result is typically a reduced pattern of co-evolution between symbiont and 370 host that can inhibit the process of metabolic and genetic integration, stalling the evolution 371 of stable interactions which are needed if a system is to move towards evolution of an 
372 organelle ${ }^{13,57-60}$. Previous studies have suggested that when selfish behaviours arise, the

373 evolution of enforcement to punish or suppress the exploitative partner can act to restore

374 cooperation $^{9,10}$. Enforcement mechanisms have been identified in diverse biological 375 systems ${ }^{61,62}$, and are argued to be one of the most effective drivers of cooperation in 376 egalitarian alliances between different species, such as that between $P$. bursaria and its algal 377 endosymbiont'. The results presented here suggest that 'RNAi collisions' between 378 endosymbiont and host, which are capable of imposing a cost to host growth for breakdown 379 of the symbiosis, could provide an emergent enforcement mechanism to discourage over380 exploitation of the endosymbiont population by the host.

381 Interestingly, the emergence of this mechanism appears to be a by-product of pre382 existing biological features that are already likely to be under strong selective pressure. For 383 instance, the widely functional RNAi system of the host ${ }^{42,43,45}$, or the conserved gene 384 repertoire and sequence composition of the host and endosymbiont transcriptomes from 385 which some 'RNAi collisions' have here been identified (including many transcripts which 386 are conditionally essential in other systems ${ }^{56}$ ). Unlike comparable mechanisms of RNA-RNA 387 interactions that have been studied in host-pathogen symbioses ${ }^{36-40}$, these 'RNAi collisions' 388 appear to be untargeted and, hence, emergent. In the aforementioned host-pathogen 389 systems, targeted RNA is passed from one partner to the other in order to modulate 390 expression of transcripts involved in virulence or resistance ${ }^{36,39,40}$. However, in order for 391 such systems to evolve, they must first exist in an untargeted form upon which selection is 392 able to act, allowing the emergence of specific RNA factors ${ }^{37,38}$. Identification of undirected 393 'RNAi collisions' in the P. bursaria - Chlorella system represents one such intermediary 394 state, emergent in nature and untargeted, upon which sustained cellular interaction 395 coupled with the potential for host-symbiont conflict could drive the selection of targeted 396 RNA-RNA interactions.

We therefore propose that 'RNAi collisions' represent a putative mechanism to discourage over-exploitation of the endosymbiont population by the host. Here we have used the example of mass endosymbiont digestion in response to drug treatment to 400 simulate this effect in the extreme. In natural interactions between $P$. bursaria and its algal 401 endosymbiont, such a cost would only need to occur in the drastic occurrence of mass 402 endosymbiont digestion in order to drive stability of the interaction. Importantly, the 
endosymbiotic algal population within P. bursaria is largely composed of closely related or

404 clonal lineages ${ }^{11-13}$, and as such, the fate of the algal population should be considered as a

405 collective unit. This cost need only act to supress large-scale, rapid destruction by the host in

406 order to drive the maintenance of a surviving subsection of the endosymbiont population.

407 Previous studies have demonstrated how $P$. bursaria is capable of manipulating

408 endosymbiont load in response to varying light conditions to better suit its own ends ${ }^{27,28}$,

409 however, in these examples, reduction of endosymbiont number through digestion is slow

410 and partial. By providing a system that selects against rapid and near-complete digestion of

411 the endosymbiont population, 'RNAi collisions' effectively buffer the nascent endosymbiotic

412 interaction against total breakdown. We suggest that this has allowed the relationship to be

413 maintained across time and varying ecological conditions, even in the event of host-

414 symbiont conflict ${ }^{27,28}$ and fluctuating endosymbiont numbers ${ }^{14,26,27}$. As an alternate route to

415 conflict resolution that avoids partner switching, we propose that such a mechanism would

416 facilitate greater co-evolution between endosymbiont and host. Over time, this would allow

417 the metabolic and genetic integration that drives the formation of obligate symbioses to

418 become manifest. We therefore present 'RNAi collisions' as a new mechanism in this

419 endosymbiotic system - a factor which can promote stability in the face of conflict in an

420 emergent endosymbiotic eukaryote-eukaryote cell-cell interaction.

\section{$421 \quad$ AUTHOR CONTRIBUTIONS}

422 B.H.J., D.S.M., and T.A.R. conceived and designed the experiments. F.M. and T.A.R. 423 conceived and designed the eDicer computational analysis. B.H.J., D.S.M., and T.A.R. wrote 424 the manuscript. B.H.J., D.S.M., F.M. and G.L. conducted experimental work and analysed the 425 data. B.E.H., S.W. and J.D.E aided in conceptual and experimental design, and in conducting 426 experimental work.

\section{ACKNOWLEDGEMENTS}

428 This work was primarily supported by an EMBO YIP award and a Royal Society University 429 Research Fellowship (UF130382) and latterly by an ERC Consolidator Grant (CELL-in-CELL) to 430 T.A.R. S.W. and J.D.E. were supported by awards from the Wellcome Trust 431 (WT107791/Z/15/Z) and the Lister institute. F.M. was supported by a Donald Hill Family 432 Fellowship in Computer Science. We thank Karen Moore and the University of Exeter 433 Sequence Service for support with the various sequencing projects. We thank Éric Meyer, 
bioRxiv preprint doi: https://doi.org/10.1101/2021.04.11.439338; this version posted May 13, 2021. The copyright holder for this preprint (which

was not certified by peer review) is the author/funder, who has granted bioRxiv a license to display the preprint in perpetuity. It is made available under aCC-BY-NC-ND 4.0 International license.

434 Institut de Biologie de l'Ecole Normale Supérieure, Paris, for advice during set up of the $P$.

435 bursaria RNAi approach.

436 DECLARATION OF INTERESTS

437 The authors declare no competing interests. 


\section{FIGURES}
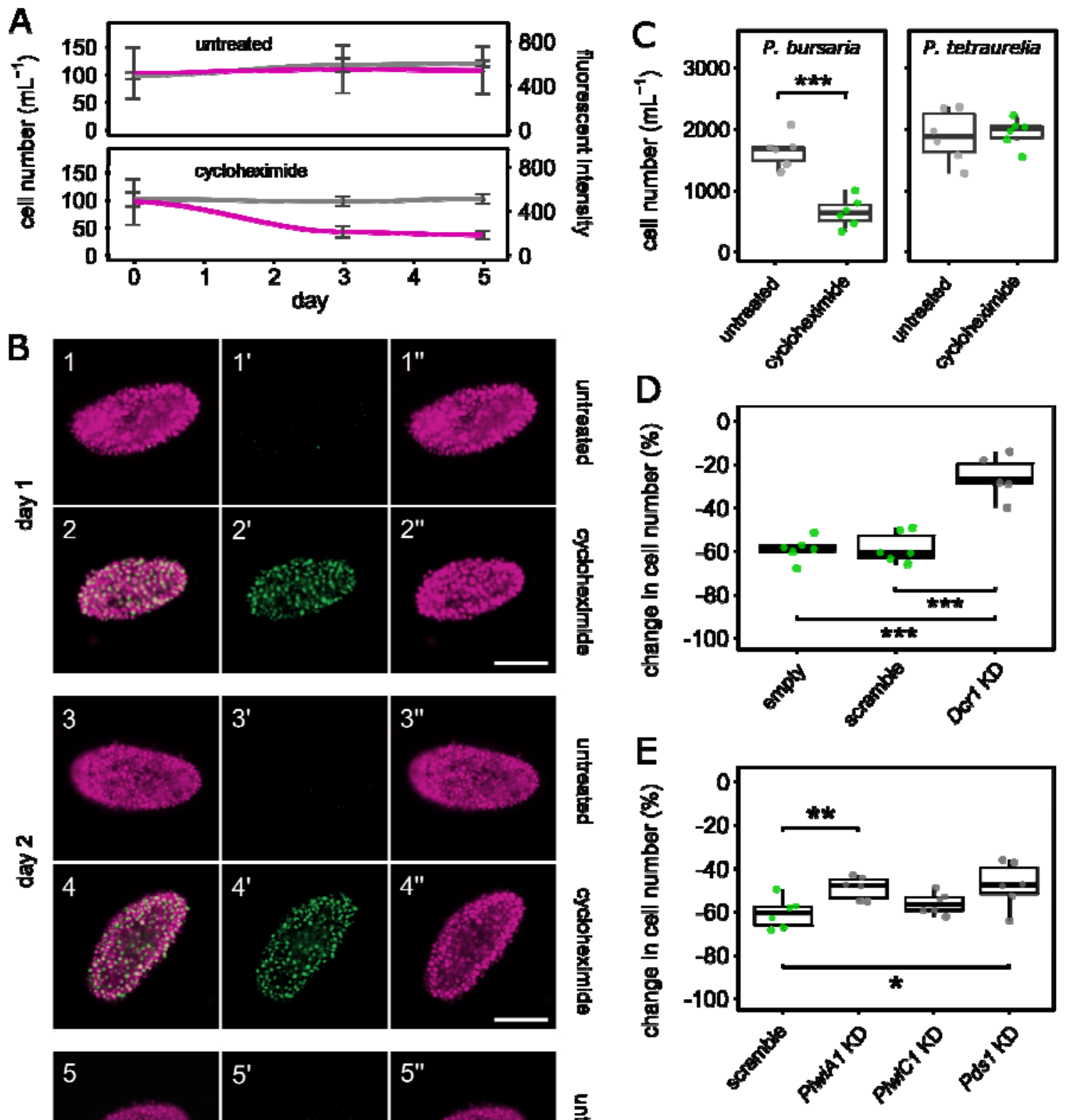

439
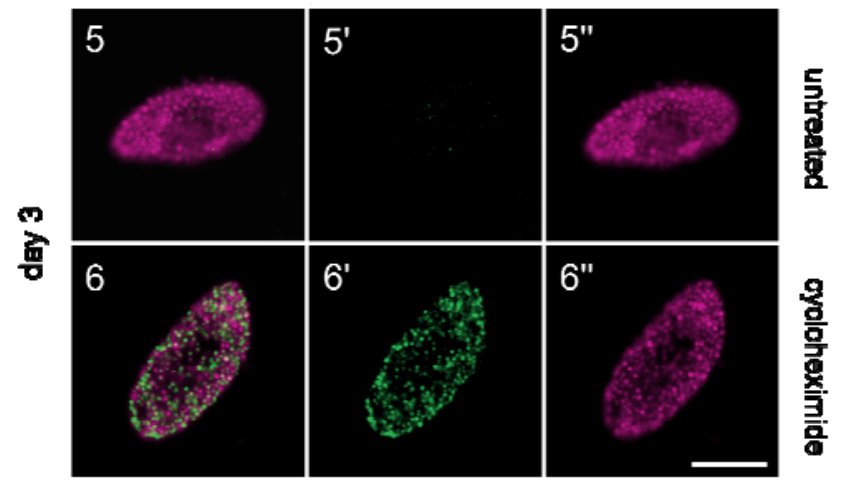
441 Figure 1. Endosymbiont digestion in P. bursaria results in an RNAi-mediated 'physiological

442 cost' to the host. (A) Paramecium cell number (grey) vs algal chlorophyll fluorescent

443 intensity (pink) in stationary phase P. bursaria cultures treated with cycloheximide (50

$\left.444 \mathrm{\mu gmL}^{-1}\right)$, compared to untreated controls. Loss of algal fluorescent intensity indicates algal

445 death in response to cycloheximide treatment. Data are represented as mean \pm SD of six

446 biological replicates. (B) Stages of endosymbiont elimination in P. bursaria cells over three

447 days of cycloheximide treatment $\left(50 \mu \mathrm{gmL}^{-1}\right)$. Algal chlorophyll fluorescence (pink) highlights

448 endosymbiotic algae within the P. bursaria cell. LysoTracker Green fluorescence (green)

449 indicates increased host lysosomal activity in response to cycloheximide treatment. Scale

450 bar $-50 \mu \mathrm{m}$. (C) Paramecium cell number in P. bursaria or P. tetraurelia cultures after 8 days

451 of treatment with cycloheximide $\left(50 \mu \mathrm{gmL}^{-1}\right.$; green), compared to untreated controls (light

452 grey). Paramecium cultures were fed with E. coli transformed with an empty RNAi vector.

453 (D) Percentage change in P. bursaria cell number in cultures treated with cycloheximide (50

$454 \mathrm{\mu gmL}^{-1}$ ), compared to untreated controls, after 12 days of feeding with $E$. coli expressing;

455 Dicer (Dcr1) dsRNA (dark grey) to induce knock-down (KD), non-hit 'scramble' dsRNA or an

456 empty vector control (green). The relative effect of Dicer dsRNA exposure indicates partial

457 rescue of $P$. bursaria culture growth retardation in response to cycloheximide induced

458 endosymbiont digestion. (E) Percentage change in P. bursaria cell number in cultures

459 treated with cycloheximide $\left(50 \mathrm{\mu gmL}^{-1}\right)$, compared to untreated controls, after 12 days of

460 feeding with E. coli expressing; PiwiA1, PiwiC1 or Pds1 dsRNA (dark grey) to induce knock-

461 down (KD), or a non-hit 'scramble' dsRNA control (green). (C-E) Feeding was conducted daily

462 for four days prior to cycloheximide treatment, and continued throughout. Boxplot data are

463 represented as max, upper quartile (Q3), mean, lower quartile (Q1) and min values of six

464 biological replicates. Individual data points are shown. Significance calculated as * $p \leq 0.05$,

$465 * * p \leq 0.01$, and $* * * p \leq 0.001$, using a generalized linear model with quasi-Poisson

466 distribution. See also Figure S3 for the raw count data used to calculate the percentage

467 change in cell number presented in 1D-E. 


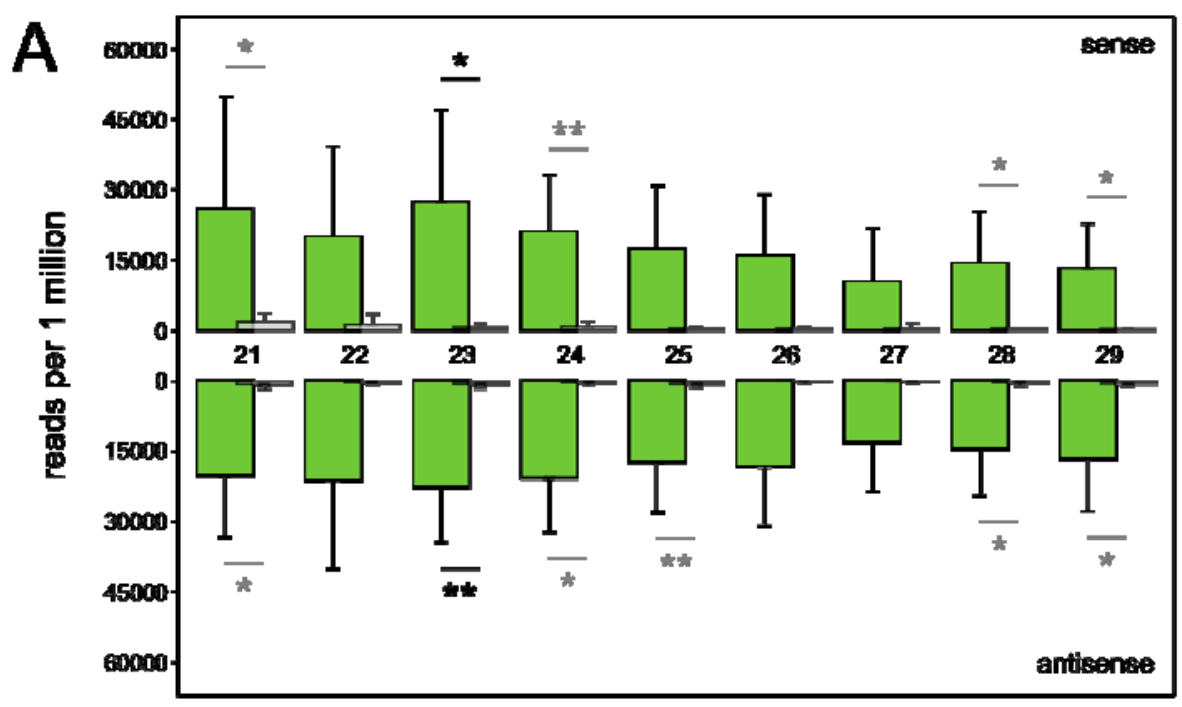

$\square$ cycloheximide

$\square$ untreated

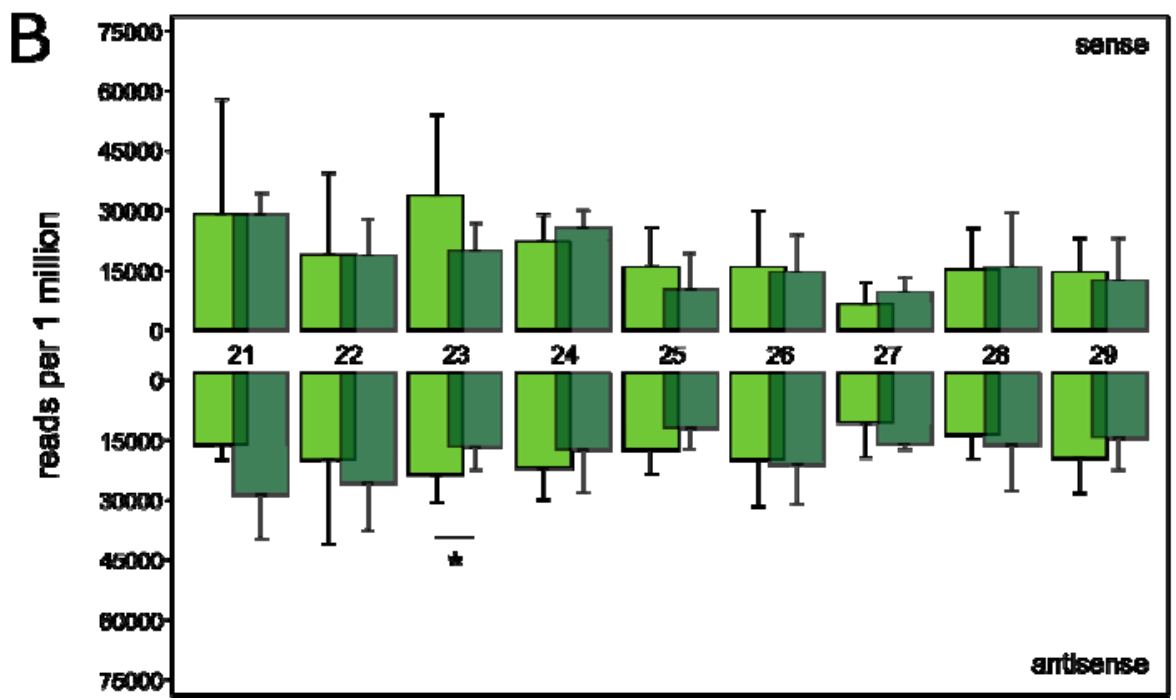

scramble

Dorr KD

470 Figure 2. Endosymbiont breakdown triggers an influx of Dicer-dependent endosymbiont-

471 derived sRNA within $\boldsymbol{P}$. bursaria. (A) Size distribution (nt) of SRNA mapped to

472 endosymbiont-derived cytoplasmic mRNA. SRNA was extracted from $P$. bursaria cultures

473 over two days (day 2 and 3) of cycloheximide treatment $\left(50 \mu \mathrm{gmL}^{-1}\right.$ ), or from untreated

474 controls. Note the relative increase in 23-nt abundance between untreated and

475 cycloheximide treated cultures. P. bursaria cultures were fed with E. coli transformed to

476 express non-hit 'scramble' dsRNA. Data are represented as mean \pm SD of six biological

477 replicates, and normalised against total endosymbiont mRNA-mapping 21-29-nt reads per

478 dataset. (B) Size distribution (nt) of sRNA mapped to endosymbiont-derived cytoplasmic

479 mRNA. SRNA was extracted from P. bursaria cultures on day 3 of cycloheximide treatment 
$480\left(50 \mu \mathrm{gmL}^{-1}\right)$. P. bursaria cultures were fed with E. coli transformed to express Dicer (Dcr1)

481 dsRNA to induce knock-down (KD), or a non-hit 'scramble' dsRNA control. Note the relative

482 increase in 23-nt abundance during cycloheximide treatment in P. bursaria cultures exposed

483 to scramble dsRNA, compared to Dicer dsRNA. Data are represented as mean \pm SD of three

484 biological replicates, and normalised against total endosymbiont mRNA-mapping 21-29-nt

485 reads per dataset. (A-B) Feeding was conducted daily for four days prior to cycloheximide

486 treatment, and continued throughout. Significance calculated as *p $\leq 0.05, * * p \leq 0.01$, and

$487 * * * p \leq 0.001$ using a generalised linear model with quasi-Poisson distribution. All curated

488 'endosymbiont' mRNA transcript bins used for sRNA mapping are available on Figshare

489 (10.6084/m9.figshare.12301736). See also Figure S4 for sRNA abundance at each individual 490 day.

491 

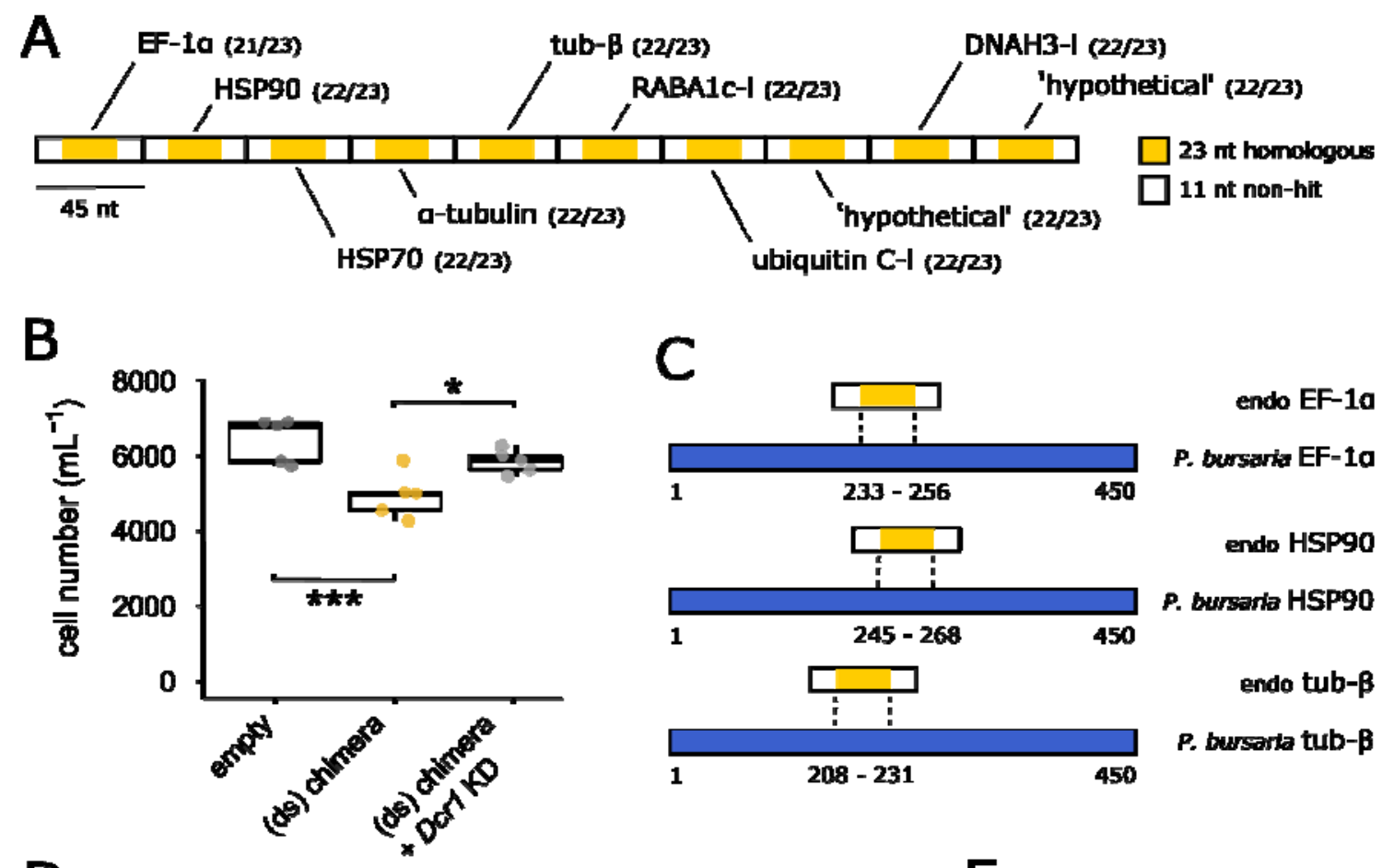

D

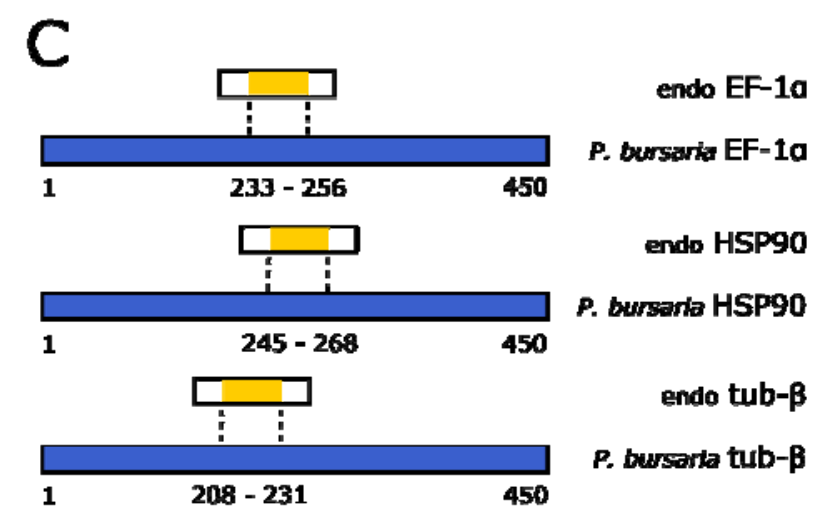

492
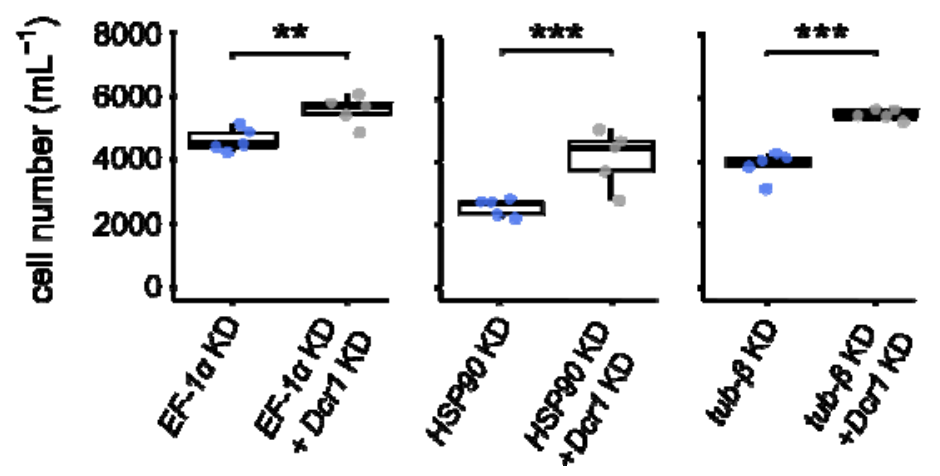

E

493

494 Figure 3. In vivo exposure to a synthetic endosymbiont-derived RNA chimera generates

495 simultaneous knock-down of homologous host genes in P. bursaria. (A) Schematic showing

496 chimeric construct design. Ten endosymbiont-derived 45-nt transcript sequences (Table S3),

497 featuring a 23-nt region with $>91 \%$ sequence identity between endosymbiont and host

498 (yellow) flanked by 11-nt of 'non-hit' algal transcript (white). Numbers in brackets denote

499 sequence identity between endosymbiont and host. (B) P. bursaria cell number after 12

500 days of feeding with $E$. coli expressing: chimera dsRNA (yellow); chimera dsRNA mixed with

501 Dicer (Dcr1) dsRNA (light grey; rescue); or an empty vector control (dark grey). See Figure S9

502 for an experimental dilution of dsRNA chimera delivery. (C) Schematic demonstrating the 
503 degree of overlap between three endosymbiont (endo) derived transcripts from the

504 chimeric construct (white/yellow) and respective 450-nt homologous region of the host ( $P$.

505 bursaria) transcript (blue). Each region of 23-nt overlap (yellow) represents putative RNAi

506 'collisions' between endosymbiont and host. (D) P. bursaria cell number after 12 days of

507 feeding with E. coli expressing: host EF1- $\alpha$, HSP9O or tub-B 450-nt dsRNA (blue) to induce

508 host knock-down (KD), compared to Dicer (Dcr1) dsRNA mixed controls (grey; rescue

509 phenotype). See also Figure S11 for individual knock-down (KD) of each of the ten broader

510 host targets of the endosymbiont-derived dsRNA chimera. (E) qPCR of mRNA extracted from

511 day 3 of chimera-RNAi feeding (3B), revealing knock-down of EF1- $\alpha, H S P 90$ and tub- 8 host

512 gene expression in $P$. bursaria in response to endosymbiont derived chimera dsRNA

513 exposure. Standardised expression of an Actin housekeeping gene was used for

514 normalisation. Data are represented as mean \pm SD of three biological replicates. These three

515 genes were assessed for knock-down as a result of endosymbiont derived chimera

516 exposure, based on evidence that directed knock-down of the wider corresponding host

517 gene led to retardation of $P$. bursaria culture growth (3D). For an extended figure showing

518 the effect of Dicer knock-down on the expression of these three host genes, see Figure S12.

519 (B/D) Multiple vector delivery was conducted at a 50:50 ratio during feeding. Boxplot data

520 are represented as max, upper quartile (Q3), mean, lower quartile (Q1) and min values of

521 five biological replicates. Individual data points are shown. Significance for boxplot data

522 calculated as $* p \leq 0.05, * * p \leq 0.01, * * * p \leq 0.001$, using a generalized linear model with

523 quasi-Poisson distribution. 

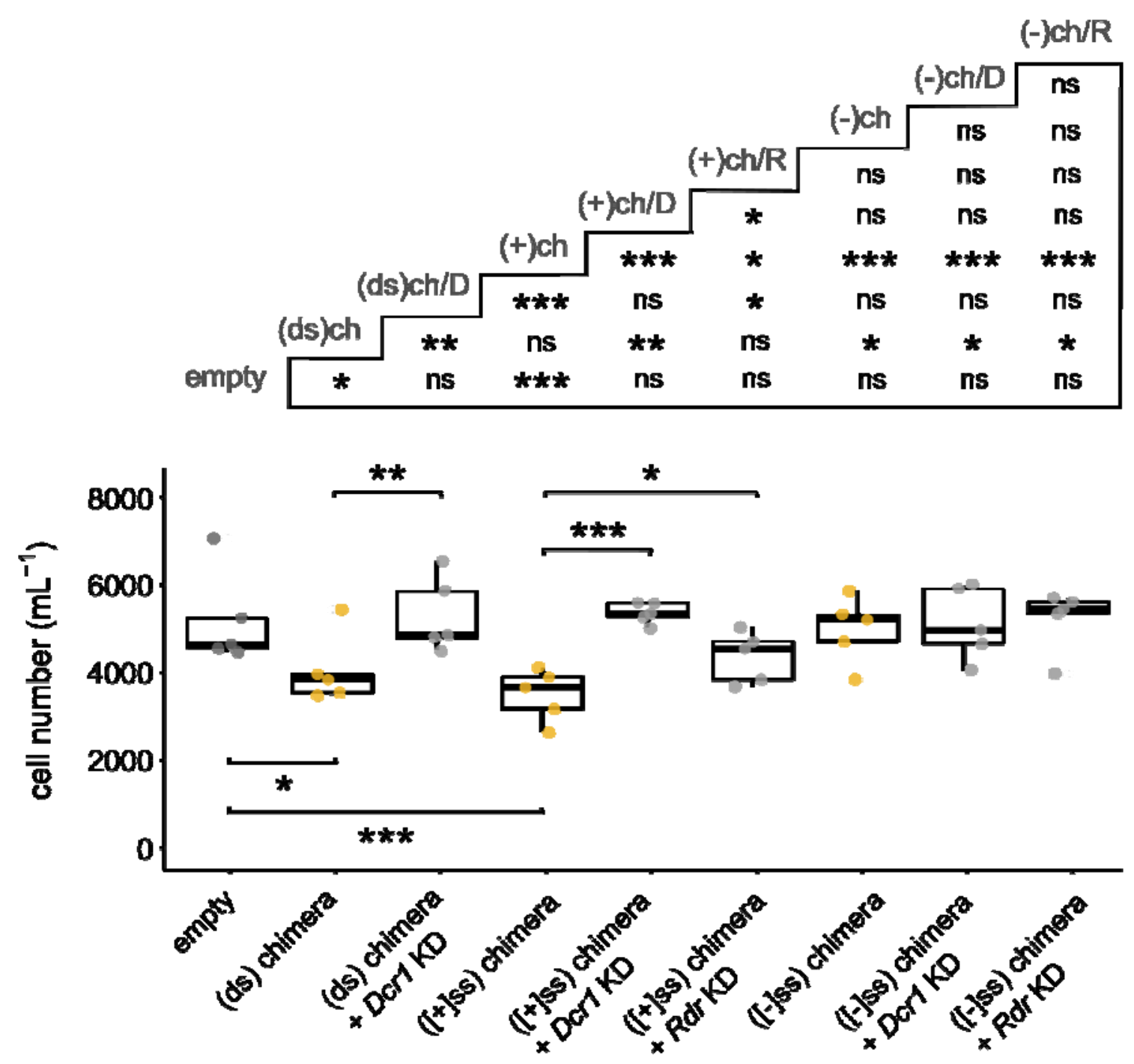

526 Figure 4. Single-stranded delivery of synthetic endosymbiont-derived RNA, analogous to

527 endosymbiont mRNA, results in a cost to $\boldsymbol{P}$. bursaria growth. $P$. bursaria cell number after

52814 days of feeding with E. coli expressing; chimera RNA in ds, [+]ss or [-]ss orientation

529 (yellow); chimera RNA in ds, [+]ss or [-]ss orientation mixed with Dicer (Dcr1) or RdRP (Rdr)

530 dsRNA (light grey; rescue); or an empty vector control (dark grey). Multiple vector delivery

531 was conducted at a 50:50 ratio during feeding. Asterisks displayed in the grid above denote

532 pairwise significance values. Boxplot data are represented as max, upper quartile (Q3),

533 mean, lower quartile (Q1) and min values of five biological replicates. All significance for

534 boxplot data calculated as * $p \leq 0.05, * * p \leq 0.01, * * * p \leq 0.001$, 'ns' no significance, using a

535 generalized linear model with quasi-Poisson distribution. 


\section{METHODS}

\section{Culture conditions and media}

538 In all RNAi experiments, Paramecium bursaria 186b (CCAP 1660/18) strain was used. For

539 experiments requiring a non-photo-endosymbiotic Paramecium system for comparison,

540 Paramecium tetraurelia nd7 strain was used. For eDicer analysis, Paramecium bursaria 186b

541 and Yad1g1N strains were used ${ }^{41,55}$.

542 Paramecium cells were cultured in New Cereal Leaf - Prescott Liquid media (NCL). NCL 543 media was prepared by adding $4.3 \mathrm{mgL}^{-1} \mathrm{CaCl}_{2} .2 \mathrm{H}_{2} \mathrm{O}, 1.6 \mathrm{mgL}^{-1} \mathrm{KCl}, 5.1 \mathrm{mgL}^{-1} \mathrm{~K}_{2} \mathrm{HPO}_{4}, 2.8$

$544 \mathrm{mgL}^{-1} \mathrm{MgSO}_{4} \cdot 7 \mathrm{H}_{2} \mathrm{O}$ to deionised water. $1 \mathrm{gL}^{-1}$ wheat bran was added, and the solution boiled 545 for 5 minutes. Once cooled, media was filtered once through Whatman Grade 1 filter paper 546 and then through Whatman GF/C glass microfiber filter paper. Filtered NCL media was 547 autoclaved at $121^{\circ} \mathrm{C}$ for 30 mins to sterilise prior to use.

$548 \quad$ NCL medium was bacterized with Klebsiella pneumoniae SMC and supplemented with $5490.8 \mathrm{mgL}^{-1} \beta$-sitosterol prior to propagation. Paramecium cells were sub-cultured 1:9 into 550 fresh bacterized NCL media once per month for Paramecium bursaria 186b, and once every 551 two weeks for Paramecium tetraurelia nd7. Paramecium cultures were maintained at $18^{\circ} \mathrm{C}$ 552 with a light-dark (LD) cycle of 12:12h.

\section{LysoTracker staining and fluorescent imaging}

554 Paramecium cells were fixed using $0.5 \%$ paraformaldehyde and incubated for 20 mins at RT.

555 Cells were then treated with $2 \mu \mathrm{M}$ LysoTracker Green DND-26 (Invitrogen) to stain acidic

556 vesicles such as the lysosome, and incubated in constant darkness for 2 hours at RT prior to 557 imaging. Stained Paramecium cells were imaged on an ImageXpress Pico Automated Cell 558 Imaging System at 10x magnification, using the Cy5 (absorbance - 630/40 nm, emission $559695 / 45 \mathrm{~nm}$ ) and FITC (absorbance $-465 / 40 \mathrm{~nm}$, emission - 525/30 nm) channels to capture 560 algal chlorophyll autofluorescence and LysoTracker Green DND-26 fluorescence 561 respectively.

\section{Gene synthesis and construct design}

563 Sequences for plasmid constructs were synthesised de novo by either Genscript or SynBio 564 Technologies, and cloned into an L4440 plasmid vector. Sequences and cloning sites for 
565 each plasmid construct are detailed in Table S1. All modified constructs were confirmed by

566 Sanger sequencing (Eurofins Genomics).

\section{$567 \quad$ RNAifeeding}

568 Paramecium was fed with E. coli transformed with an L4440 plasmid construct with paired 569 IPTG-inducible T7 promoters, facilitating targeted gene knock-down through the delivery of 570 complementary double-stranded RNA (dsRNA). L4440 plasmid constructs were transformed 571 into $E$. coli HT115 competent cells and grown overnight on LB agar (50 $\mu \mathrm{gmL}^{-1} \mathrm{Ampicillin}$ and $57212.5 \mu \mathrm{gmL}^{-1}$ Tetracycline) at $37^{\circ} \mathrm{C}$. Positive transformants were picked and grown overnight 573 in LB (50 $\mathrm{gmL}^{-1}$ Ampicillin and $12.5 \mu \mathrm{gmL}^{-1}$ Tetracycline) at $37^{\circ} \mathrm{C}$ with shaking (180 rpm).

574 Overnight pre-cultures were back-diluted 1:25 into $50 \mathrm{~mL}$ of LB (50 $\mathrm{ggmL}^{-1}$ Ampicillin and $57512.5 \mathrm{\mu gmL}^{-1}$ Tetracycline) and incubated for a further 2 hours under the same conditions, 576 until an $\mathrm{OD}_{600}$ of between 0.4 and 0.6 was reached. E. coli cultures were then supplemented 577 with $0.4 \mathrm{mM}$ IPTG to induce template expression within the L4440 plasmid, and incubated 578 for a further 3 hours under the same conditions. E. coli cells were pelleted by centrifugation 579 (3100 $\times g$ for 2 mins), washed with sterile NCL media, and pelleted once more. E. coli cells 580 were then re-suspended in $\mathrm{NCL}$ media supplemented with $0.4 \mathrm{mM}$ IPTG, $100 \mu \mathrm{gmL}^{-1}$ 581 Ampicillin, and $0.8 \mu \mathrm{gmL}^{-1} \beta$-sitosterol, and adjusted to a final $\mathrm{OD}_{600}$ of 0.1 .

582 Paramecium cells were pelleted by gentle centrifugation in a 96-well plate (10 mins 583 at $800 \times g$ ), taking care not to disturb the cell pellet by leaving $50 \mu$ l of supernatant, and re584 suspended 1:4 into $200 \mu$ of induced E. coli culture media (to make $250 \mu$ l total). Feeding 585 was conducted daily for up to 14 days using freshly prepared bacterized media.

586 Single-stranded construct design and confirmation

587 To create a version of L4440 which expressed only single-stranded (ssRNA), L4440 was

588 digested with Kpnl and Pvull (Promega), gel-purified (Wizard SV Gel and PCR Clean-Up 589 System, Promega) and blunted using PfuUltra HF DNA polymerase (Agilent Technologies).

590 The blunt vector was re-ligated using T4 DNA ligase (Thermo Scientific) and confirmed by 591 sequencing (Eurofins Genomics), generating plasmid pDM004 which contains only a single 592 T7 promoter. Fragments were then excised from their respective $\mathbf{4} 4440$ plasmids, or 593 amplified by PCR (Q5 Polymerase; New England Biolabs) to swap the restriction sites, 594 digested, and ligated into pDM004 to generate plasmids containing inserted fragments in 
595 sense [+] or antisense [-] orientation. Generated plasmids were transformed into HT115 E.

596 coli for use in RNAi feeding experiments.

597 To confirm that these plasmid constructs generated only ssRNA, cultures of $E$. coli 598 HT115 containing pDM005-1 ([-]ssRNA chimera) or pDM005-2 ([+]ssRNA chimera) were 599 grown overnight at $37^{\circ} \mathrm{C}, 180 \mathrm{rpm}$ in LB supplemented with $50 \mu \mathrm{gmL}^{-1}$ ampicillin and 12.5 $600 \mathrm{\mu gmL}^{-1}$ tetracycline. Cultures were diluted 1:25 in fresh medium and grown to an $\mathrm{OD}_{600}$ of 601 0.4-0.6. Expression was then induced with $400 \mu \mathrm{M}$ IPTG for $3 \mathrm{hrs}$, after which $1 \mathrm{~mL}$ of 602 culture was pelleted by centrifugation for 2 mins at 3,100 $\times g$. RNA was extracted using an 603 RNeasy Mini Kit (Qiagen), following the manufacturer's protocol for Total RNA Purification 604 from Animal Cells. $20 \mu$ l of RNA was then treated with $10 \mu \mathrm{gmL}^{-1}$ RNase A (Sigma-Aldrich) for 6051 hour at $30^{\circ} \mathrm{C}$ in the presence of $300 \mathrm{mM} \mathrm{NaCl}$ (stabilising dsRNA and allowing RNase $A$ to 606 degrade only ssRNA ${ }^{63}$ ). A separate aliquot was left untreated, with $300 \mathrm{mM} \mathrm{NaCl}$ added to 607 facilitate precipitation. All samples were then extracted with 1:1 phenol:chloroform and 608 precipitated with 2 volumes of ethanol. Pellets were washed twice with $80 \%$ ethanol and re609 suspended in nuclease-free water. RNA samples were then cleared of residual genomic DNA 610 using the TURBO DNA-free Kit (Ambion), following the manufacturer's protocol for routine 611 DNase treatment. RT-PCR was then performed using the Qiagen OneStep RT-PCR kit 612 following the manufacturer's instructions, with $0.5 \mu \mathrm{L}$ template RNA and $0.6 \mu \mathrm{M}$ each 613 primer (pDM005_RT_F: 5'-ACTTCAATGATTCGCAGCGG-3' and pDM005_RT_R: 5'614 AAGTAGCTGCTGTTCTCGGT-3'), generating an 85-nt PCR product. Cycling conditions were as 615 detailed in the manufacturer's protocol (30 cycles), with $1 \mathrm{~min}$ annealing at $50^{\circ} \mathrm{C}$. PCR 616 products were then resolved on a $2 \%$ agarose gel to assess for the presence/absence of 617 amplification in each sample (Figure S11).

\section{$618 \quad$ qPCR analysis}

619 RNA was extracted from P. bursaria 186b for gene expression analysis after three days of 620 RNAi feeding. Paramecium cells ( $\sim 10^{3}$ per culture) were pelleted by gentle centrifugation 621 (800 $\mathrm{g}$ for 10 mins), snap-frozen in liquid nitrogen, and stored at $-80^{\circ} \mathrm{C}$. RNA extraction was 622 performed using TRIzol reagent (Invitrogen), following the manufacturer's protocol after re623 suspending each pellet in $900 \mu \mathrm{l}$ TRIzol reagent. RNA was precipitated using GlycoBlue Co624 precipitant (Invitrogen) to aid RNA pellet visualisation, and then cleared of residual DNA 
625 using the TURBO DNA-free Kit (Ambion), following the manufacturer's protocol for routine

626 DNase treatment.

627 RNA was reverse transcribed into single stranded cDNA using the SuperScript ${ }^{\circledR}$ III First-

628 Strand Synthesis SuperMix (Invitrogen), following the manufacturer's protocol. Quantitative 629 PCR (qPCR) was performed in a StepOnePlus Real-Time PCR system (Thermo Fisher

630 Scientific). Reaction conditions were optimised using a gradient PCR, with a standard curve 631 determined using 10-fold dilutions of P. bursaria cDNA: EF-1 $\alpha$ (slope: -3.353 ; $\mathrm{R}^{2}$ : 0.999; 632 efficiency 98.740\%), HSP90 (slope: -3.319; R2: 0.998; efficiency 100.131\%), tub- $\beta$ (slope: 633 3.378; $R^{2}$ : 0.992; efficiency 97.692\%), and actin (slope: -3.349; $R^{2}$ : 0.983; efficiency $63498.866 \%)$, using StepOne software v2.3. Each $20 \mu \mathrm{L}$ reaction contained $10 \mu \mathrm{L}$ PowerUp SYBR 635 Green Master Mix (Thermo Fisher Scientific), $500 \mathrm{nM}$ each primer (300 nM for tub- $\beta$ ) and 1 $636 \mu \mathrm{L}(50 \mathrm{ng}) \mathrm{cDNA}$. Each reaction was performed in duplicate for each of 3 biological 637 replicates, alongside a 'no-RT' (i.e. non-reverse transcribed $\mathrm{RNA}$ ) control to detect any 638 genomic DNA contamination. Cycling conditions were as follows: UDG activation, 2 mins at $63950^{\circ} \mathrm{C}$ and DNA polymerase activation, 2 mins at $95^{\circ} \mathrm{C}$, followed by 40 cycles of 15 secs, $95^{\circ} \mathrm{C}$ 640 and $1 \mathrm{~min}$ at $55-65^{\circ} \mathrm{C}\left(E F-1 \alpha\left(58^{\circ} \mathrm{C}\right), \operatorname{HSP} 90\left(58^{\circ} \mathrm{C}\right)\right.$, tub- $\beta\left(57^{\circ} \mathrm{C}\right)$ and actin $\left.\left(58^{\circ} \mathrm{C}\right)\right)$. Primers 641 pairs for each reaction are listed in Table S5. Each reaction was followed by melt-curve 642 analysis, with a $60-95^{\circ} \mathrm{C}$ temperature gradient $\left(0.3^{\circ} \mathrm{C} \mathrm{s}^{-1}\right)$, ensuring the presence of only a 643 single amplicon, and ROX was used as a reference dye for calculation of $C_{T}$ values. $C_{T}$ values 644 were then used to calculate the change in gene expression of the target gene in RNAi 645 samples relative to control samples, using a derivation of the $2^{-\Delta \Delta C T}$ algorithm ${ }^{64}$.

\section{6 sRNA isolation and sequencing}

647 Total RNA for sRNA sequencing was extracted from P. bursaria (or free-living algal) cultures 648 using TRIzol reagent (Invitrogen), as detailed above. To isolate sRNA from total RNA, 649 samples were size separated on a denaturing 15\% TBE-UREA polyacrylamide gel. Gels were 650 prepared with a $15 \mathrm{~mL}$ mix with final concentrations of $15 \%$ Acrylamide/Bis (19:1), $8 \mathrm{M}$ 651 UREA, TBE ( $89 \mathrm{mM}$ Tris, $89 \mathrm{mM}$ Borate, $2 \mathrm{mM}$ EDTA), and the polymerisation started by the 652 addition of $150 \mu \mathrm{L}$ 10\% APS (Sigma-Aldrich) and $20 \mu \mathrm{L}$ TEMED (Sigma-Aldrich). Gels were 653 pre-equilibrated by running for 15 mins ( $200 \mathrm{~V}, 30 \mathrm{~mA})$ in TBE before RNA loading. The 654 ladder mix consisted of $500 \mathrm{ng}$ ssRNA ladder (50-1000nt, NEB\#N0364S), and 5-10 ng of each $65521 \& 26-n t$ RNA oligo loaded per lane. The marker and samples were mixed with 2X RNA 
656 loading dye (NEB) and heat denatured at $90{ }^{\circ} \mathrm{C}$ for 3 mins before snap cooling on ice for 2

657 min prior to loading. Blank lanes were left between samples/replicates to prevent cross-

658 contamination during band excision. Gels were then run for 50 mins ( $200 \mathrm{~V}, 30 \mathrm{~mA}$ ).

659 Once run, gels were stained by shaking (60 rpm) for 20 mins at RT in a $40 \mathrm{~mL}$ TBE 660 solution containing $4 \mu \mathrm{LSYBR}^{\circledR}$ Gold Nucleic Acid Gel Stain. Bands of the desired size range 661 ( 15-30 nt) were visualised under blue light, excised and placed into a $0.5 \mathrm{~mL}$ tube pierced 662 at the bottom by a 21-gauge needle, resting within a $1.5 \mathrm{~mL}$ tube, and centrifuged $(16,000 \mathrm{x}$ $663 g$ for $1 \mathrm{~min}$ ). $400 \mu \mathrm{L}$ of RNA elution buffer (1M Sodium acetate $\mathrm{pH} 5.5$ and $1 \mathrm{mM}$ EDTA) was 664 added to the $1.5 \mathrm{~mL}$ tube containing centrifuged gel slurry, and the empty $0.5 \mathrm{~mL}$ tube 665 discarded. Gel slurry was manually homogenized until dissolved using a $1 \mathrm{~mL}$ sterile plunger 666 and incubated at RT for 2 hours with shaking at 1,400 rpm.

667 Solutions containing RNA elution buffer and gel slurry were transferred to a Costar 668 Spin-X $0.22 \mu \mathrm{m}$ filter column and centrifuged $(16,000 \times g$ for $1 \mathrm{~min})$. The filter insert 669 containing acrylamide was discarded. $1 \mathrm{~mL}$ of $100 \% \mathrm{EtOH}$ was added to each solution, 670 alongside $15 \mu \mathrm{g}$ of GlycoBlue ${ }^{\mathrm{TM}}$ Coprecipitant (Invitrogen) to aid sRNA pellet visualisation, 671 and stored overnight at $-80^{\circ} \mathrm{C}$ to precipitate. Precipitated solutions were centrifuged at $4{ }^{\circ} \mathrm{C}$ $672(12,000 \times g$ for 30 mins), and the supernatant discarded. sRNA pellets were washed with 500 $673 \mu \mathrm{L}$ of cold $70 \% \mathrm{EtOH}\left(12,000 \times g\right.$ for 15 mins at $\left.4^{\circ} \mathrm{C}\right)$, and air dried in a sterile PCR hood for 67410 mins, before re-suspending in $15 \mu \mathrm{L}$ of RNAse-free water and storage at $-80^{\circ} \mathrm{C}$.

\section{SRNA-seq and read processing}

676 sRNA concentrations were determined using an Agilent 2100 Bioanalyzer, following the 677 Agilent Small RNA kit protocol, and all samples matched to $0.7 \mathrm{ngmL}^{-1}$ prior to sequencing. 678 Library preparation and subsequent RNA-seq was performed for 54 samples using 50-bp 679 paired-end, rapid run across four lanes on an Illumina HiSeq 2500, yielding 120-150 million 680 paired-end reads per lane ( 9-11 million paired-end reads per sample).

681 The raw paired-end reads from the RNA-seq libraries were trimmed using Trim Galore 682 in order to remove barcodes (4-nt from each 3'- and 5'- end) and SRNA adaptors, with 683 additional settings of a phred-score quality threshold of 20 and minimum length of 16-nt. 684 Result were subsequently checked with FastQC.

685 Assigning sRNAs to the algal endosymbiont transcript bins 
686 Trimmed reads were mapped against the 'endosymbiont' dataset of assembled transcripts

687 using the HISAT2 alignment program with default settings. Post-mapping, the BAM files

688 were processed using SAMTOOLS and a set of custom scripts 689 (https://github.com/guyleonard/paramecium) to produce a table of mapped read 690 accessions and their respective read lengths. Using these exported count tables, of mapped 691 reads per read length, transcripts with $>10$ hits for read lengths between 21-25 nt were 692 searched using reciprocal BLASTX against the NCBI non-redundant ' $n r$ ' proteins sequence 693 database, in order to assign taxonomic identity to each transcript. This allowed filtering of 694 the main 'endosymbiont' dataset into subsets corresponding to either: algal cytoplasmic 695 mRNA, algal cytoplasmic rRNA, algal plastid RNA or algal mitochondrial RNA; host RNA 696 contamination; bacterial RNA contamination; or vector RNA contamination. All identified 697 algal cytoplasmic rRNA (28 transcripts), plastid RNA (56 transcripts) or mitochondrial RNA 698 (18 transcripts) sequences were sorted into new datasets representing each RNA species. 699 Host (5 transcripts) and bacterial (34 transcripts) contamination were sorted into respective 700 'host' or 'bacterial' datasets. Vector (1 transcript) and all unidentifiable sequences (20 701 transcripts) were separated into a dataset labelled as 'other'. All remaining transcripts with $702>10$ sRNA hits for read sizes $21-25 \mathrm{nt}$ in the original dataset now represented putative algal 703 mRNA transcripts (i.e. for 23-nt reads this represented 3,659 sRNA reads mapping to 148 704 transcripts). In this algal mRNA dataset, we also included transcripts that fell below the $>10$ 705 sRNA hit threshold for manual curation, and which therefore may correspond to reads 706 mapping to all of the above bins (i.e. for 23-nt reads this represented 1,949 reads mapping 707 to 605 transcripts in total, of which 1,408 reads were mapping to 468 transcripts which were 708 not manually curated, so could be affected by contamination). This manual binning process 709 was carried out in order to double check the original automated binning post transcriptome 710 assembly, and to check for possible chimeric host-algal transcript sequences produced as a 711 by-product of cDNA synthesis and transcriptome sequencing and assembly. This process 712 collectively allowed accurate segregation of the existing 'endosymbiont' transcript bin into 713 discrete subsets based on RNA 'species'.

714 Using the HISAT2 alignment program with default settings, trimmed reads were 715 mapped once more against the newly filtered algal mRNA, rRNA, plastid and mitochondrial 716 datasets from above. Count tables of mapped reads per read length were once again 
717 generated from the BAM files, and used to plot a size distribution of 21-29 nt endosymbiont

718 derived sRNA abundance per RNA species. Size distributions of sRNA abundance for each

719 sample were plotted using the R programming language packages; tidyverse, grid.extra and 720 ggplot2 in R Studio.

721 eDicer methods for identifying putative RNA-RNA interactions

722 To predict putative mRNA-mRNA interactions using eDicer

723 (https://github.com/fmaguire/eDicer), both the 'host' and 'endosymbiont' transcript bins 724 processed from transcriptome data for $P$. bursaria Yad1g1 $\mathrm{N}^{55}$ were further filtered to 725 minimise the risk of false positives for host and endosymbiont cross-comparisons. For the 726 'host' bins, any transcript with $>90 \%$ ID BLASTN hit to the following genome assemblies 727 were removed: Chlamydomonas reinhardtii cc503 cw92 mt, Chlamydomonas reinhardtii v3, 728 Chlorella sorokiniana 1228 v2, Chlorella sorokiniana DOE1412 v3, or Chlorella sorokiniana 729 utex1230 lanl v2 assemblies from Los Alamos National Labs Greenhouse genome database. 730 Similarly, for the 'endosymbiont' bins, any transcript with $>90 \%$ ID BLASTN hit to the 731 following ParameciumDB genome assemblies were removed: $P$. biaurelia V1-4 v1, $P$. 732 caudatum 43c3d v1, P. bursaria MAC 110224 v1, P. decaurelia MAC 223 v1, P. dodecaurelia 733 MAC 274 v1, P. jenningsi MAC M v1, P. novaurelia MAC TE v1, P. octaurelia K8 CA1, $P$. 734 quadecaurelia MAC NiA v1, P. primaurelia Ir4-2 v1, P. tetraurelia MAC 51 with and without 735 IES, or P. tetraurelia MAC. Additionally, the reference (refseq) genome of Escherichia coli 736 MG1655 (NCBI acc.: NZ_CP012868.1) and Klebsiella pneumoniae HS11286 (NCBI acc.: 737 NC_016845.1) were used as 'food' comparison datasets.

738 The filtered 'endosymbiont' transcript bin, E. coli CDS and K. pneumoniae CDS were 739 decomposed into all possible 21-23 nt reads with jellyfish v2.2.10 and aligned with 95\% 740 identity to the 'host' bin using Bowtie v1.2.3 (recommended for short alignments). These in741 silico RNA-RNA interaction simulations were performed using a wrapper tool we created 742 named eDicer (v1.0.0) (https://github.com/fmaguire/eDicer). The number of distinct 743 aligning k-mers (i.e. putative interactions of unique sequences) for each pair of bins were 744 then normalised by dividing by the total number of distinct k-mers in both bins, and $x 100$, 745 to calculate a Jaccard Index \% (i.e. normalised set similarity).

746 To predict putative 'lethal' RNA-RNA interactions, a separate analysis was conducted 747 using a subset of each dataset that was putatively homologous to a yeast 'lethal' gene 
748 database ${ }^{56}$. This 'lethal' dataset contained genes known to be conditionally essential in

749 Saccharomyces cerevisiae. These putative 'lethal' homologues for each dataset were

750 identified using a tBLASTx search of the yeast 'lethal' database ${ }^{56}$ with a gathering threshold

751 set at 1 e-10 with a minimum of $50 \%$ sequence identity. Once curated, each 'lethal' dataset

752 was subject to eDicer analysis as described above.

753 To predict putative rRNA-rRNA interactions using eDicer, a further analysis was 754 conducted using a dataset consisting of full-length ribosomal RNA (rRNA) clusters for 755 Microactinium conductrix $[\mathrm{NCBI} \text { acc.: } \mathrm{ASM} 224581 \mathrm{v} 2]^{65}$, K. pneumoniae $[\mathrm{NCBI}$ acc.: 756 NC_016845.1] $]^{66}$, E. coli [NCBI acC.: NZ_CP012868.1], P. bursaria Yad1g1N ${ }^{55}$ and P. bursaria 757 186b. Once again, each dataset was subject to eDicer analysis as described above. All in758 silico RNA-RNA interaction predictions were plotted using the $\mathrm{R}$ programming language 759 packages; tidyverse, grid.extra and ggplot2 in R Studio.

\section{Manual curation of additional host transcript bins}

761 In order to identify host transcripts that could be impacted by putative RNA-RNA 762 interactions as a result of endosymbiont derived sRNA exposure, trimmed Illumina reads for 763 all sRNA sequencing samples were mapped to the 'endosymbiont mRNA' transcript dataset. 764 Resulting mapping files were filtered with custom scripts 765 (https://github.com/guyleonard/paramecium) producing tables of mapped hits with their 766 respective read lengths. Reads from all tables, of length 23-nt only, were then extracted with 767 SEQTK (resulting in 3,690 total). A BLASTn search of these 23-nt endosymbiont reads was 768 conducted against the 'host' transcript dataset, to identify host transcripts with $\geq 95 \%$ 769 identity over a 23-nt region. This resulted in three candidate host transcripts. These three 770 host transcripts were searched using BLASTx against the NCBI non-redundant 'nr' protein 771 database, resulting in one transcript with $100 \%$ sequence identity to both endosymbiont 772 and host HSP90. For the remaining two transcripts, SNPs present in the identified 23-nt 773 mapped sequence indicate that these are putative host sequences with 2-nt of mismatch 774 compared to the respective endosymbiont sequence, and were therefore unlikely to 775 represent a product of RNA-RNA interactions between endosymbiont and host as a result of 776 endosymbiont derived 23-nt sRNA exposure.

777 In order to identify host transcripts that could be classified as 'non-hit' transcripts (i.e. 778 host transcripts sharing a low level of sequence identity with algal transcripts over 23-nt 
779 regions), the 'host' dataset was searched using an organism specific BLASTn (Chlorellaceae

780 [NCBI: taxid35461]) against the $\mathrm{NCBI}$ nucleotide ' $\mathrm{nr} / \mathrm{nt}$ ' database with a minimum

781 expectation of $1 \mathrm{e}-05$. Any host transcripts sharing $>20-n t$ sequence identity with algal

782 transcripts over a 23-nt region were rejected. This process was repeated until a dataset of

78320 'non-hit-algal' host transcripts were identified. These putative 'non-hit-algal' host

784 transcripts were then searched using BLASTx against the NCBI non-redundant 'nr' protein

785 database, to confirm that these 20 transcripts were derived from Paramecium and were not

786 bacterial contamination.

\section{DATA AND SOFTWARE AVAILABILITY}

788 The raw reads generated during sRNA sequencing are available on the NCBI Sequence Read

789 Archive (accessions: SAMN14932981, SAMN14932982). All other datasets are available on

790 Figshare (https://doi.org/10.6084/m9.figshare.c.4978160.v1), under the relevant headings.

791 Custom scripts for sRNA read processing (https://github.com/guyleonard/paramecium,

792 https://doi.org/10.5281/zenodo.4638888) and eDicer comparative analysis

793 (https://github.com/fmaguire/eDicer, https://doi.org/10.5281/zenodo.4659378) are

794 available on GitHub and archived within the Zenodo repository.

\section{BIBLIOGRAPHY}

796 1. Archibald, J. M. Endosymbiosis and eukaryotic cell evolution. Curr. Biol. 25, R911-921

$797 \quad$ (2015).

798 2. Baurain, D. et al. Phylogenomic evidence for separate acquisition of plastids in

799 Cryptophytes, Haptophytes, and Stramenopiles. Molecular biology and evolution 27,

$800 \quad 1698-709(2010)$.

801 3. Keeling, P. J. The number, speed, and impact of plastid endosymbioses in eukaryotic

802 evolution. Annual Review of Plant Biology 64, 583-607 (2013).

803 4. Archibald, J. M. The puzzle of plastid evolution. Curr Biol 19, R81-8 (2009).

804 5. Howe, C. J., Barbrook, A. C., Nisbet, R. E. R., Lockhart, P. J. \& Larkum, A. W. D. The

805 origin of plastids. Philos Trans R Soc Lond B Biol Sci 363, 2675-2685 (2008). 
806 6. Timmis, J. N., Ayliffe, M. A., Huang, C. Y. \& Martin, W. Endosymbiotic gene transfer:

807 organelle genomes forge eukaryotic chromosomes. Nat. Rev. Genet. 5, 123-135 (2004).

808 7. Gray, M. W. Mitochondrial evolution. Cold Spring Harb Perspect Biol 4, (2012).

809 8. Moran, N. A. Symbiosis as an adaptive process and source of phenotypic complexity.

$810 \quad$ Proc. Natl. Acad. Sci. U.S.A. 104 Suppl 1, 8627-8633 (2007).

811 9. Ågren, J. A., Davies, N. G. \& Foster, K. R. Enforcement is central to the evolution of

$812 \quad$ cooperation. Nat Ecol Evol 3, 1018-1029 (2019).

813 10. West, S. A., Kiers, E. T., Simms, E. L. \& Denison, R. F. Sanctions and mutualism

814 stability: why do rhizobia fix nitrogen? Proceedings of the Royal Society of London.

815 Series B: Biological Sciences 269, 685-694 (2002).

816 11. Achilles-Day, U. E. \& Day, J. G. Isolation of clonal cultures of endosymbiotic green

817 algae from their ciliate hosts. J Microbiol Methods 92, 355-7 (2013).

818 12. Hoshina, R. \& Kusuoka, Y. DNA Analysis of algal endosymbionts of ciliates reveals the

819 state of algal integration and the surprising specificity of the symbiosis. Protist 167, 174$820 \quad 184(2016)$.

821 13. Zagata, P., Greczek-Stachura, M., Tarcz, S. \& Rautian, M. The evolutionary relationships 822 between endosymbiotic green algae of Paramecium bursaria syngens originating from 823 different geographical locations. Folia Biol (Krakow) 64, 47-54 (2016).

824 14. Kodama, Y. \& Fujishima, M. Cycloheximide induces synchronous swelling of perialgal 825 vacuoles enclosing symbiotic Chlorella vulgaris and digestion of the algae in the ciliate $826 \quad$ Paramecium bursaria. Protist 159, 483-94 (2008).

827 15. Kodama, Y. \& Fujishima, M. Symbiotic Chlorella variabilis incubated under constant

828 dark conditions for 24 hours loses the ability to avoid digestion by host lysosomal 829 enzymes in digestive vacuoles of host ciliate Paramecium bursaria. FEMS Microbiol. $830 \quad$ Ecol. 90, 946-955 (2014). 
831 16. Omura, G. et al. A bacteria-free monoxenic culture of Paramecium bursaria: its growth

832 characteristics and the re-establishment of symbiosis with Chlorella in bacteria-free

833 conditions. Jpn J Protozool 37, (2004).

834 17. Tanaka, M. et al. Complete elimination of endosymbiotic algae from Paramecium

835 bursaria and its confirmation by diagnostic PCR. Acta Protozool 41, 255-261 (2002).

836 18. Brown, J. A. \& Nielsen, P. J. Transfer of photosynthetically produced carbohydrate from

837 endosymbiotic Chlorellae to Paramecium bursaria. J. Protozool. 21, 569-570 (1974).

838 19. Esteban, G. F., Fenchel, T. \& Finlay, B. J. Mixotrophy in ciliates. Protist 161, 621-641

$839 \quad$ (2010).

840 20. Johnson, M. D. Acquired phototrophy in ciliates: a review of cellular interactions and

$841 \quad$ structural adaptations. J Eukaryot Microbiol 58, 185-95 (2011).

842 21. Kato, Y. \& Imamura, N. Amino acid transport systems of Japanese Paramecium

843 symbiont F36-ZK. Symbiosis 47, 99-107 (2009).

844 22. Kato, Y. \& Imamura, N. Effect of sugars on amino acid transport by symbiotic Chlorella.

$845 \quad$ Plant Physiol Biochem 46, 911-7 (2008).

846 23. Kawakami, H. \& Kawakami, N. Behavior of a virus in a symbiotic system, Paramecium

847 bursaria-Zoochlorella. The Journal of Protozoology 25, 217-225 (1978).

848 24. Parker, R. C. Symbiosis in Paramecium bursaria. Journal of Experimental Zoology 46,

$849 \quad 1-12(1926)$.

850 25. Ziesenisz, E., Reisser, W. \& Wiessner, W. Evidence of de novo synthesis of maltose

851 excreted by the endosymbiotic Chlorella from Paramecium bursaria. Planta 153, 481-

$852485(1981)$.

853 26. Kodama, Y. \& Fujishima, M. Infectivity of Chlorella species for the ciliate Paramecium

854 bursaria is not based on sugar residues of their cell wall components, but on their ability 
855 to localize beneath the host cell membrane after escaping from the host digestive vacuole

856 in the early infection process. Protoplasma 231, 55-63 (2007).

857 27. Lowe, C. D., Minter, E. J., Cameron, D. D. \& Brockhurst, M. A. Shining a light on

858 exploitative host control in a photosynthetic endosymbiosis. Curr Biol 26, 207-211

859 (2016).

860 28. Sørensen, M. E. S. et al. The role of exploitation in the establishment of mutualistic

861 microbial symbioses. FEMS Microbiol Lett 366, (2019).

862 29. Sørensen, M. E. S., Wood, A. J., Cameron, D. D. \& Brockhurst, M. A. Rapid

863 compensatory evolution can rescue low fitness symbioses following partner-switching.

864 bioRxiv 2020.11.06.371401 (2020) doi:10.1101/2020.11.06.371401.

865 30. Axelrod, R. \& Hamilton, W. D. The evolution of cooperation. Science 211, 1390-1396

$866 \quad$ (1981).

867 31. Foster, K. R. \& Wenseleers, T. A general model for the evolution of mutualisms. J. Evol.

$868 \quad$ Biol. 19, 1283-1293 (2006).

869 32. Frank, S. A. Foundations of Social Evolution. (Princeton University Press, 1998).

870 33. Herre, E. A., Knowlton, N., Mueller, U. G. \& Rehner, S. A. The evolution of mutualisms:

871 exploring the paths between conflict and cooperation. Trends in Ecology \& Evolution 14,

$872 \quad 49-53(1999)$.

873 34. Szathmáry, E. \& Smith, J. M. The major evolutionary transitions. Nature 374, 227-232

$874 \quad$ (1995).

875 35. West, S. A., Fisher, R. M., Gardner, A. \& Kiers, E. T. Major evolutionary transitions in 876 individuality. PNAS 112, 10112-10119 (2015).

877 36. Buck, A. H. et al. Exosomes secreted by nematode parasites transfer small RNAs to

878 mammalian cells and modulate innate immunity. Nat Commun 5, 5488 (2014). 
879 37. Guo, Z., Li, Y. \& Ding, S. W. Small RNA-based antimicrobial immunity. Nat Rev

$880 \quad$ Immunol 19, 31-44 (2019).

881 38. Huang, C. Y., Wang, H., Hu, P., Hamby, R. \& Jin, H. Small RNAs - big players in plant-

882 microbe interactions. Cell Host Microbe 26, 173-182 (2019).

883 39. Wang, M. et al. Bidirectional cross-kingdom RNAi and fungal uptake of external RNAs

884 confer plant protection. Nat Plants 2, 16151 (2016).

885 40. Weiberg, A. et al. Fungal small RNAs suppress plant immunity by hijacking host RNA

886 interference pathways. Science 342, 118-23 (2013).

887 41. Jenkins, B. H. et al. Characterization of the RNA-interference pathway as a tool for

888 reverse genetic analysis in the nascent phototrophic endosymbiosis, Paramecium

$889 \quad$ bursaria. Royal Society Open Science 8, 210140.

890 42. Carradec, Q. et al. Primary and secondary siRNA synthesis triggered by RNAs from food

891 bacteria in the ciliate Paramecium tetraurelia. Nucleic Acids Res 43, 1818-33 (2015).

892 43. Galvani, A. \& Sperling, L. RNA interference by feeding in Paramecium. Trends Genet

$893 \quad \mathbf{1 8}, 11-2(2002)$.

894 44. Karunanithi, S. et al. Exogenous RNAi mechanisms contribute to transcriptome

895 adaptation by phased siRNA clusters in Paramecium. Nucleic Acids Res 47, 8036-8049

$896 \quad$ (2019).

897 45. Marker, S., Carradec, Q., Tanty, V., Arnaiz, O. \& Meyer, E. A forward genetic screen

898 reveals essential and non-essential RNAi factors in Paramecium tetraurelia. Nucleic

$899 \quad$ Acids Res 42, 7268-80 (2014).

900 46. Marker, S., Le Mouël, A., Meyer, E. \& Simon, M. Distinct RNA-dependent RNA

901 polymerases are required for RNAi triggered by double-stranded RNA versus truncated

902 transgenes in Paramecium tetraurelia. Nucleic Acids Res 38, 4092-107 (2010). 
903 47. Karunanithi, S. et al. Feeding exogenous dsRNA interferes with endogenous sRNA

904 accumulation in Paramecium. DNA research $\square$ : an international journal for rapid

905 publication of reports on genes and genomes $\mathbf{2 7}$, (2020).

906 48. Cerutti, H., Ma, X., Msanne, J. \& Repas, T. RNA-mediated silencing in algae: biological

907 roles and tools for analysis of gene function ?. Eukaryot Cell 10, 1164-1172 (2011).

908 49. Hoehener, C., Hug, I. \& Nowacki, M. Dicer-like enzymes with sequence cleavage

$909 \quad$ preferences. Cell 173, 234-247.e7 (2018).

910 50. Ahmed, F. \& Raghava, G. P. S. Designing of highly effective complementary and

911 mismatch siRNAs for silencing a gene. PLoS One 6, (2011).

912 51. Du, Q., Thonberg, H., Wang, J., Wahlestedt, C. \& Liang, Z. A systematic analysis of the

913 silencing effects of an active siRNA at all single-nucleotide mismatched target sites.

$914 \quad$ Nucleic Acids Res 33, 1671-1677 (2005).

915 52. Jackson, A. L. et al. Widespread siRNA “off-target" transcript silencing mediated by

916 seed region sequence complementarity. RNA 12, 1179-1187 (2006).

917 53. Doench, J. G., Petersen, C. P. \& Sharp, P. A. siRNAs can function as miRNAs. Genes

918 Dev 17, 438-442 (2003).

919 54. Ameres, S. L., Martinez, J. \& Schroeder, R. Molecular basis for target RNA recognition

$920 \quad$ and cleavage by human RISC. Cell 130, 101-112 (2007).

921 55. Kodama, Y. et al. Comparison of gene expression of Paramecium bursaria with and

922 without Chlorella variabilis symbionts. BMC Genomics 15, 183 (2014).

923 56. Cotton, J. A. \& McInerney, J. O. Eukaryotic genes of archaebacterial origin are more

924 important than the more numerous eubacterial genes, irrespective of function.

925 Proceedings of the National Academy of Sciences 107, 17252-17255 (2010).

926 57. Fujishima, M. \& Kodama, Y. Endosymbionts in Paramecium. Eur J Protistol 48, 124-37

$927 \quad$ (2012). 
928 58. Matthews, J. L. et al. Partner switching and metabolic flux in a model cnidarian-

929 dinoflagellate symbiosis. Proc Biol Sci 285, (2018).

930 59. Matsuura, Y. et al. Recurrent symbiont recruitment from fungal parasites in cicadas.

$931 \quad$ PNAS 115, E5970-E5979 (2018).

932 60. Husnik, F. \& McCutcheon, J. P. Repeated replacement of an intrabacterial symbiont in

933 the tripartite nested mealybug symbiosis. PNAS 113, E5416-E5424 (2016).

934 61. Hickey, D. A. Selfish DNA: a sexually-transmitted nuclear parasite. Genetics 101, 519-

$935 \quad 531(1982)$.

936 62. Kiers, E. T., Rousseau, R. A., West, S. A. \& Denison, R. F. Host sanctions and the

937 legume-rhizobium mutualism. Nature 425, 78-81 (2003).

938 63. Hansen, D. R., Van Alfen, N. K., Gillies, K. \& Powell, W. A. Naked dsRNA associated

939 with hypovirulence of Endothia parasitica is packaged in fungal vesicles. Journal of

$940 \quad$ General Virology, 66, 2605-2614 (1985).

941 64. Zhang, J. D., Biczok, R. \& Ruschhaupt, M. ddCt: The ddCt Algorithm for the Analysis of

942 Quantitative Real-Time PCR (qRT-PCR). (Bioconductor version: Release (3.11), 2020).

943 doi:10.18129/B9.bioc.ddCt.

944 65. Arriola, M. B. et al. Genome sequences of Chlorella sorokiniana UTEX 1602 and

945 Micractinium conductrix SAG 241.80: implications to maltose excretion by a green alga.

$946 \quad$ The Plant Journal 93, 566-586 (2018).

947 66. Liu, P. et al. Complete genome sequence of Klebsiella pneumoniae subsp. pneumoniae

948 HS11286, a multidrug-resistant strain isolated from human sputum. J. Bacteriol. 194,

$949 \quad$ 1841-1842 (2012).

950

951 